
\title{
25 Research Square \\ Effect of Laser on Double-Arc Physical Characteristics in Pulsed Laser Induced Double-TIG Welding
}

Liming Liu

Dalian University of Technology

\section{Xinkun Xu}

Dalian University of Technology

Guomin Xu

Dalian University of Technology

Zhaodong Zhang ( $\nabla$ skyezzd@dlut.edu.cn )

Dalian University of Technology

\section{Research Article}

Keywords: Hybrid welding, Pulsed laser, Double-TIG, Energy utilization efficiency, Energy density, Coupling discharge

Posted Date: May 17th, 2021

DOl: https://doi.org/10.21203/rs.3.rs-513454/v1

License: (c) (i) This work is licensed under a Creative Commons Attribution 4.0 International License. Read Full License

Version of Record: A version of this preprint was published at The International Journal of Advanced Manufacturing Technology on November 24th, 2021. See the published version at https://doi.org/10.1007/s00170-021-08277-8. 


\title{
Effect of Laser on Double-Arc Physical Characteristics in Pulsed Laser Induced Double-TIG Welding
}

\author{
Liming Liu (刘黎明), Xinkun Xu (徐信坤), Guomin Xu (徐国敏), Zhaodong Zhang (张兆栋)
}

Key Laboratory of Liaoning Advanced Welding and Joining Technology, School of Materials Science and Engineering, Dalian University of Technology, Dalian, 116024, China.

Corresponding Author / E-mail: skyezzd@ dlut.edu.cn, TEL: +86-411-8470 7817, FAX: +86-411-8470 7817

\begin{abstract}
In order to improve the energy utilization efficiency of double-TIG arc, based on the synchronous induction of pulsed laser to double-arc, the coupling-enhanced discharge phenomenon between double-arc is studied in this paper. The energy utilization efficiency of pulsed laser induced double-arc is quantitatively analyzed. The physical characteristics of coupling double-arc are studied based on high-speed camera and spectral diagnosis technology. The physical model is established to discuss the particle migration during the coupling discharge, characterizing the movement state of electrons. The results show that Dla (horizontal distance between the tungsten electrode tip and laser beam axis) has a significant effect on the energy utilization efficiency of the heat source. With the increase of Dla, there are three interaction relationships between the double-arc plasma and keyhole plasma: Mutual interference, Coupling-enhanced discharge and No interaction. A suitable Dla can realize the synchronous induction of laser to double-arc plasma, forming a coupling double-arc, the heat source has a higher energy utilization efficiency. The migration of particles in double-arc can be divided into four stages: Double-arc free discharge stage, Laser-induced initial stage, Laser-induced contraction stage and Laser-induced stable stage. Under the induction of laser, the double-arc forms a common compressed conductive channel. The energy density of coupling double-arc reaches 9.3 times that of traditional double-arc, and the total electron kinetic energy increases by $38 \%$ compared with traditional double-arc.
\end{abstract}

Keywords: Hybrid welding; Pulsed laser; Double-TIG; Energy utilization efficiency; Energy density; Coupling discharge.

\section{Introduction}

Low-energy consumption and high-efficiency welding technology has become a research hot spot and an important goal in the field of welding. For decades, traditional arc welding has been widely used in various industries due to its unique advantages (strong adaptability, simple operation, low equipment cost, etc.) [1, 2]. With higher requirements for production efficiency in industry, new welding methods have been developed. Double-TIG arc welding is a stable and efficient welding method, which can significantly improve the welding speed and deposition rate. TIG arc is the best welding method for active metal materials (titanium alloy, magnesium alloy, aluminum alloy and high strength steel) due to its low sensitivity to hot cracking [3, 4]. Similar improved welding methods have many forms, such as double-sided double-arc welding [5], double-electrode MIG welding [6], double-wire three-arc welding [7], and arc wire TIG welding [8]. Compared with the traditional single arc, the double-arc generates two arcs on two insulated electrodes and acts in the same molten pool, which improves the total heat input in the welding pool and changes the heat distribution. The current direction in the two arcs is the same, producing electromagnetic field. The electromagnetic force between the charged wires causes the two arcs to attract each other. The interaction between the arcs affects the welding quality and stability [9]. Schwedersky et al. found that the double-TIG welding process has better performance than the traditional single-TIG, using a total current of 600A at a speed of $1.2 \mathrm{~m} / \mathrm{min}$ to obtain a well-shaped weld [10]. Qin et al. also reached a similar conclusion [11]. Liu et 
al. achieved high deposition rate of aluminum alloy parts by using double-electrode gas metal arc (DE-GMA) based additive manufacturing (AM) [12]. Rossini et al. pointed out that when the arc is stable, the welding speed of Tandem process is $20 \%$ higher than that of GMAW [13]. The smaller arc pressure of double-arc can effectively avoid undercut and hump defects in high-speed welding and improve welding speed [14]. However, increasing the upper limit of welding current is the key to realize high efficiency double-arc welding. The physical properties of doublearc heat source have not changed: the low energy density and low energy utilization rate, which are the main problem faced by double-arc welding technology, which limits its rapid development and application.

In addition, with the advantages of low heat input, small welding deformation and high energy density, laser welding has rapidly become an important processing method in the welding field $[15,16]$. Due to the problems of high butt joint accuracy, poor weld bridging ability, as well as poor stability in the welding of high reflectivity materials [17], laser-arc hybrid welding technology has attracted extensive attention of researchers since it was proposed in 1979 [18-21]. The preheating effect of arc plasma on the plate greatly boosts the absorption rate of laser energy and improves the welding effect of laser on high reflection materials [22]. Shinn et al. found that in the laserGMAW hybrid welding of titanium alloys, the laser attracts the cathode spots of the arc to stabilize the arc. When the welding speed is increased, the laser power needs to be increased to stabilize the arc [23]. Hu et al. found that laser-induced vaporized metal particles replaced the shielding gas particles in the arc when using low-power laserTIG hybrid welding of low carbon steel, causing a composition change of the arc plasma and promoting the stability of the arc discharge [24]. Li et al. conducted research on laser-GMAW hybrid welding steel and found that when the laser point is located at the edge of the arc, an ionization channel will be formed between the laser and the arc. When the laser point is located at the center of the arc, the arc conductive space shrinks and energy concentrated [25]. Based on the synergetic effect between laser and single arc, the coupling-enhanced discharge between two TIG arcs induced by laser may be an effective way to improve the arc energy utilization efficiency. However, the physical properties of laser induced double-TIG plasma are not clear. Previous work has initially revealed the synchronous induction mechanism of the pulsed laser to the double-arc plasma [26]. It is found that when the combing electromagnetic force between the keyhole current and the two arc currents is greater than the stiffness of the double-arc, the synchronous induction phenomenon can be achieved. Since the welding arc is the source of heat and force in welding process, it is necessary to make an in-depth study on the root cause of the energy utilization efficiency improvement of coupling arc under the laser pulse induction.

Due to the characteristics of low melting point, low boiling point and low ionization energy of magnesium alloy [27], the effect of double-arc coupling-enhanced discharge can be amplified, which is beneficial to the observation of experimental phenomena. Therefore, magnesium alloy is selected for welding test. This study quantitatively analyzed the energy utilization efficiency of pulsed laser induced double-TIG arc. The internal characteristics (dynamic behavior of plasma and keyhole, electron temperature and electron density) of double-arc plasma before and after laser induction were studied in detail by using high-speed camera and spectrum analyzer, and the energy density distribution of coupling double-arc was calculated. A physical model was established to analyze the particle migration process during the coupling discharge, characterizing the movement state of electrons and revealing the physical essence of the energy utilization efficiency improvement of laser induced double-TIG arc.

\section{MATERIALS AND METHODS}

As shown in Fig. 1, the laser-double TIG hybrid welding system consists of a pulsed Nd: YAG laser power supply and two independent OTC AEP-500P TIG welding power supplies. The wavelength of the laser beam is $1064 \mathrm{~nm}$, and a circular spot with a diameter of $0.6 \mathrm{~mm}$ is focused on the workpiece by a convex lens with a focal length of $150 \mathrm{~mm}$. Laser beam is perpendicular to the surface of the workpiece. The rated average power of the laser 
is $1000 \mathrm{~W}$, and the pulse energy is controlled by the excitation current and pulse width of the pulsed laser. Doublearc is generated by two TIG welding torches which are close to each other. The welding torches are respectively equipped with specially processed ceramic nozzles to ensure that the distance between two tungsten electrode tip (Daa) can be adjusted freely. Two TIG welding torches are placed on the same side of the laser beam and symmetrically distributed on the X-Z plane where the laser beam axis is. The angle between welding torches is about $15^{\circ}$, the angle between the torch and the workpiece about $45^{\circ}$. Daa and horizontal distance between the tungsten electrode tip and laser beam axis (Dla) could be adjusted respectively by the micrometer translation stage, realizing the paraxial hybrid of arc and laser. Argon with the purity of $99.99 \%$ is used as protecting gas, and the flow rate of protecting gas through the torch nozzle is set as $12 \mathrm{~L} / \mathrm{min}$.

In order to observe the plasma and keyhole behavior of laser-induced double-arc, as shown in Fig. 1, highspeed camera and diode laser generator are used to monitor the welding arc and weld pool surface. The high-speed camera is placed perpendicular to the welding direction. A narrow-band filter with a central wavelength of 809.5 $\mathrm{nm}$ and full width at half maximum $9.2 \mathrm{~nm}$ is installed in front of the high-speed camera lens to collect the light emission of Ar atoms in the arc. In addition, in order to clearly observe the keyhole behavior, diode laser power supply is needed to assist lighting, and the output power is $0-40 \mathrm{~W}$. The wavelength of the auxiliary light source is $808 \mathrm{~nm}$, as shown in Fig. 2. A circular bright area with a diameter of $30 \mathrm{~mm}$ is formed on the surface of the plate, which is able to cover the whole welding pool. The maximum power density of the circular spot is about $5.66 \mathrm{w} / \mathrm{cm}^{2}$. The acquisition frequency of the camera is set at $1000 \mathrm{frames} / \mathrm{s}$.

The SP-2556 emission spectrometer manufactured by Princeton Company was used to collect spectral information of arc plasma in the welding process, as shown in Fig. 1. The optical fiber probe transmits the acquired plasma optical emission signal to the spectrometer. Under the transformation of computer software (WinSpec/32), the intensity - wavelength curve is formed. The spectral information in the hybrid welding process is shown in Fig. 2. In order to reduce the error, the temperature of the vacuum chamber inside the spectrometer was cooled to $-70^{\circ} \mathrm{C}$ before data collection. The wavelength range, exposure time and resolution of spectral information acquisition can be set by computer software. In this study, a 300 grove / $\mathrm{mm}$ grating with a resolution of $0.128 \mathrm{~nm}$ was used to collect the spectral information of the arc plasma within the wavelength range of $200 \mathrm{~nm}$ to $1000 \mathrm{~nm}$, which was used to calculate the electron temperature. A 2400 grove / $\mathrm{mm}$ grating with a resolution of $0.011 \mathrm{~nm}$ was used to collect the information of a single spectral line and calculate the electron density of the arc plasma. The exposure time and data readout time are set to $20 \mathrm{~ms}$ and $13.4 \mathrm{~ms}$ respectively. The processing methods of spectral information (about the calculation of electron temperature and electron density) can be found in references [28-30]. The physical parameters of plasma spectral lines can be found from NIST (National Institute of Standards and Technology) database [31].

The experiment was carried out at atmospheric pressure and room temperature. The $6 \mathrm{~mm}$ AZ31B $\mathrm{Mg}$ alloy plate is used as the welding workpiece, and the chemical composition of Mg alloy is shown in Table 1. The oxide film and grease on the plate surface were cleaned before welding. During the welding process, the workpiece moves parallel to the x-axis, the laser beam and TIG arc are in a fixed position, and remain relatively static with the highspeed camera and optical fiber probe of spectrograph. The stability of plasma information acquisition is ensured. After welding, using an acid solution $(5 \% \mathrm{HCl}+95 \%$ alcohol) to corrode the welded joints and observing the morphology of the joints. 


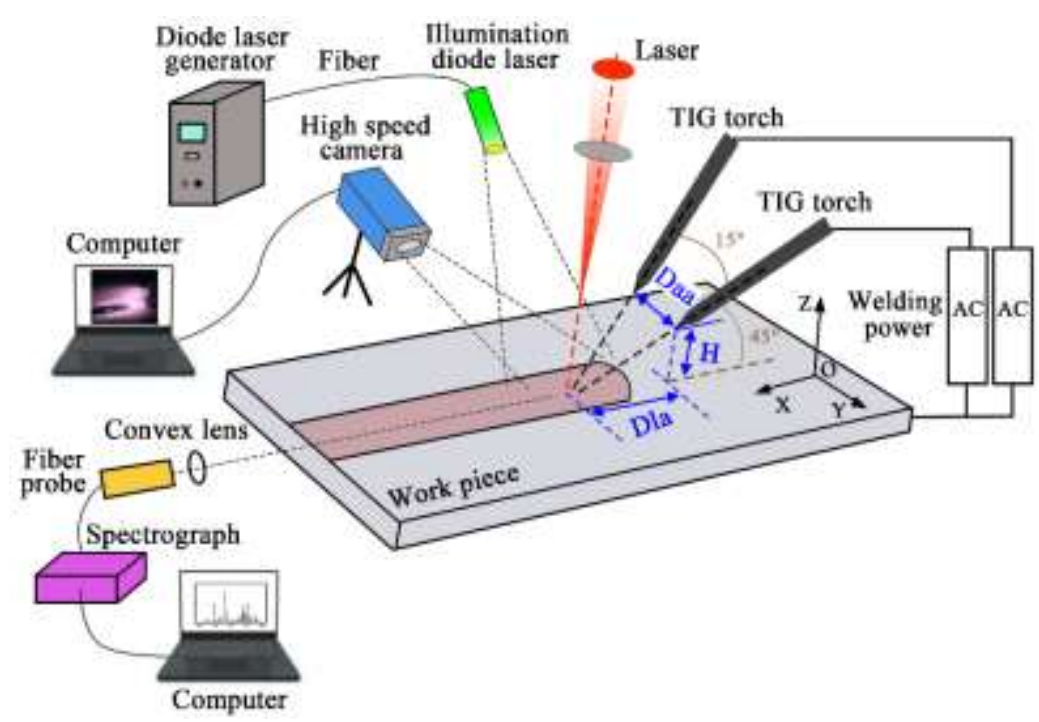

Fig. 1 Laser-double TIG hybrid welding and diagnosis system

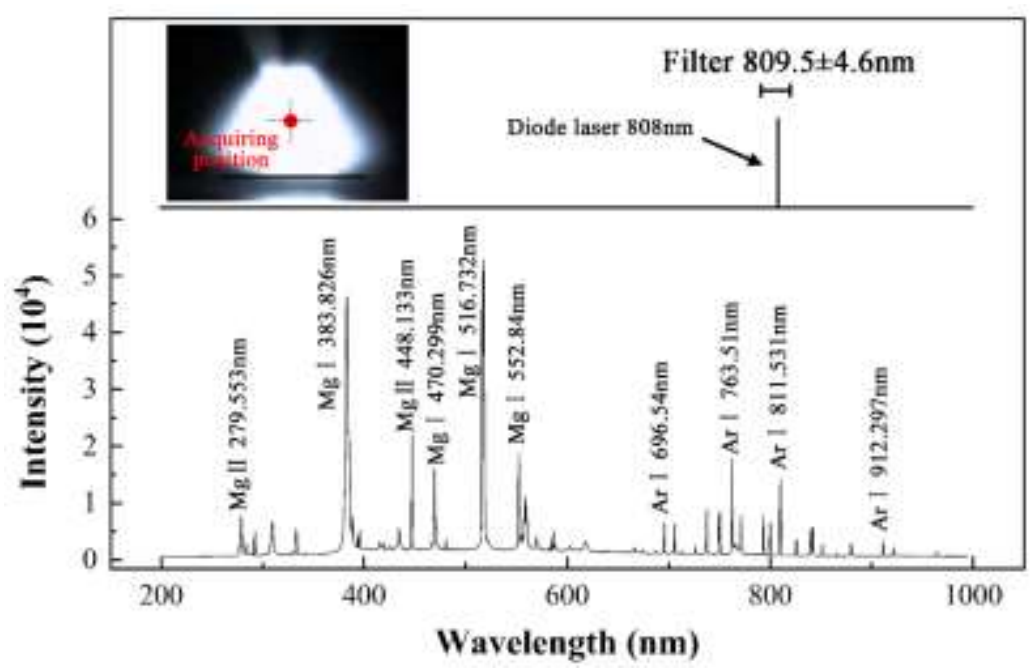

Fig. 2 Hybrid welding spectral lines and optical filter

Table 1. Chemical composition (wt\%) of AZ31B Mg alloy.

\begin{tabular}{ccccccc}
\hline Alloy & $\mathrm{Al}$ & $\mathrm{Zn}$ & $\mathrm{Mn}$ & $\mathrm{Si}$ & $\mathrm{Cu}$ & $\mathrm{Mg}$ \\
\hline AZ31B & $2.5 \sim 3.5$ & $0.7 \sim 1.3$ & $0.2 \sim 1.0$ & $<0.05$ & $<0.01$ & Bal. \\
\hline
\end{tabular}

\section{Results}

\subsection{Quantitative analysis of energy utilization efficiency}

In order to study the energy utilization efficiency of pulsed laser induced double-TIG arc, welding experiments were carried out on $6 \mathrm{~mm}$ thick AZ31B magnesium alloy sheet. The related research of laser-single arc hybrid welding shows that the parameter Dla has a great influence on the interaction between laser and arc plasma [32]. Therefore, while keeping the laser power, welding speed and other welding parameters unchanged, a series of process tests were carried out by adjusting Dla and arc current. The welding parameters are shown in Table 2 . The cross-sectional morphology of joints under different welding processes is shown in Fig. 1. The energy utilization efficiency can be characterized by the change of melting energy of hybrid welding compared with single laser welding and traditional double-arc welding. During welding, the melting energy is the energy of melting the sheet 
provided by the heat source, which can be expressed by Formula 1 [33]:

$$
E_{\mathrm{m}}=(v S) \rho\left(\int_{\mathrm{T}_{0}}^{\mathrm{T}_{\mathrm{m}}} c \mathrm{~d} T+H\right)
$$

Where $E_{\mathrm{m}}$ is the melting energy, $v S$ is the volume of molten metal per unit time, $v$ is the welding speed, $S$ is the cross-sectional area of joint, $\rho$ is the material density, $T$ is the temperature, $\mathrm{T}_{0}$ is the room temperature, $\mathrm{T}_{\mathrm{m}}$ is the melting point of material, $c$ is the specific heat capacity and $H$ is the latent heat of fusion.

Therefore, the melting energy of hybrid welding, traditional double-arc welding, and single laser welding can be expressed as:

$$
\begin{aligned}
E_{\mathrm{LDA}} & =\left(v S_{\mathrm{LDA}}\right) \rho\left(\int_{\mathrm{T}_{0}}^{\mathrm{T}_{\mathrm{m}}} c \mathrm{~d} T+H\right) \\
E_{\mathrm{DA}} & =\left(v S_{\mathrm{DA}}\right) \rho\left(\int_{\mathrm{T}_{0}}^{\mathrm{T}_{\mathrm{m}}} c \mathrm{~d} T+H\right) \\
E_{\mathrm{L}} & =\left(v S_{\mathrm{L}}\right) \rho\left(\int_{\mathrm{T}_{0}}^{\mathrm{T}_{\mathrm{m}}} c \mathrm{~d} T+H\right)
\end{aligned}
$$

Where $E_{\mathrm{LDA}}, E_{\mathrm{DA}}, E_{\mathrm{L}}$ are the melting energy of hybrid welding, traditional double-arc welding, and single laser welding, respectively, and $S_{\mathrm{LDA}}, S_{\mathrm{DA}}, S_{\mathrm{L}}$ are the cross-sectional area of joints during hybrid welding, traditional double-arc welding, and single laser welding, respectively.

This research is carried out under the same experimental conditions (base material type, temperature and welding speed), so $\int_{\mathrm{T}_{0}}^{\mathrm{T}_{\mathrm{m}}} c \mathrm{~d} T+H$ can be regarded as a constant. Based on the above discussion, a dimensionless parameter of melting energy $\psi$, namely the increment of melting energy, is introduced to quantitatively analyze the energy utilization efficiency of laser induced double-TIG arc.

$$
\psi=\frac{E_{\mathrm{LDA}}-\left(E_{\mathrm{DA}}+E_{\mathrm{L}}\right)}{E_{\mathrm{DA}}+E_{\mathrm{L}}} \times 100 \%=\frac{S_{\mathrm{LDA}}-\left(S_{\mathrm{DA}}+S_{\mathrm{L}}\right)}{S_{\mathrm{DA}}+S_{\mathrm{L}}} \times 100 \%
$$

In the formula, the cross-sectional area of joints can be measured in Fig. 3. The larger the value of $\psi$ is, the higher the energy utilization efficiency is.

Fig. 4 shows the calculated melting energy increment of heat source under different parameter combinations. It can be seen from the figure that when the arc current is fixed, as Dla increases, the value of $\psi$ increases first and then decreases. The maximum value of $\psi$ depends on Dla at different arc current levels. As the arc current changes from $80 \mathrm{~A}+80 \mathrm{~A}$ to $110 \mathrm{~A}+110 \mathrm{~A}$, the value of Dla corresponding to the maximum value of $\psi$ increases from $3 \mathrm{~mm}$ to $5 \mathrm{~mm}$. Therefore, the Dla corresponding to the maximum melting energy increment increases with the increase of arc current level. It is worth noting that when the arc current is $100 \mathrm{~A}+100 \mathrm{~A}$ and Dla is $4 \mathrm{~mm}$, the maximum value of $\psi$ reaches $177.5 \%$, which is higher than the maximum $\psi$ value of other two current levels, indicating that the heat source has higher energy utilization efficiency at this time.

Table 2. Laser-double TIG hybrid welding parameters.

\begin{tabular}{cccc}
\hline Parameters & Values & Parameters & Values \\
\hline Laser pulse energy Q/J & 15 & Daa $/ \mathrm{mm}$ & 1 \\
Dla/mm & $1 \sim 6$ & Double-arc current/A & $80+80,100+100$, \\
& & $\mathrm{H} / \mathrm{mm}$ & $110+110$ \\
Laser pulse frequency $/ \mathrm{Hz}$ & 30 & Welding speed $/ \mathrm{mm} \cdot \mathrm{min}^{-1}$ & 2 \\
Laser pulse duration $w_{\mathrm{p}} / \mathrm{ms}$ & 3 & & 800 \\
\hline
\end{tabular}




\begin{tabular}{|c|c|c|c|c|c|c|c|c|}
\hline \multirow{2}{*}{$\begin{array}{l}\text { Double-are } \\
\text { current }\end{array}$} & \multicolumn{6}{|c|}{ Dla/mm } & \multirow{2}{*}{ double-asc } & \multirow{2}{*}{ laser } \\
\hline & 1 & 2 & 3 & 4 & 5 & 6 & & \\
\hline $80 \mathrm{~A}+80 \mathrm{~A}$ & & & & & & & 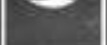 & \\
\hline $100 \mathrm{~A}+100 \mathrm{~A}$ & & & & & & & 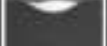 & 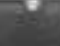 \\
\hline $110 \mathrm{~A}+110 \mathrm{~A}$ & & & & & & & & \\
\hline
\end{tabular}

Fig. 3 Joint morphology under different welding processes

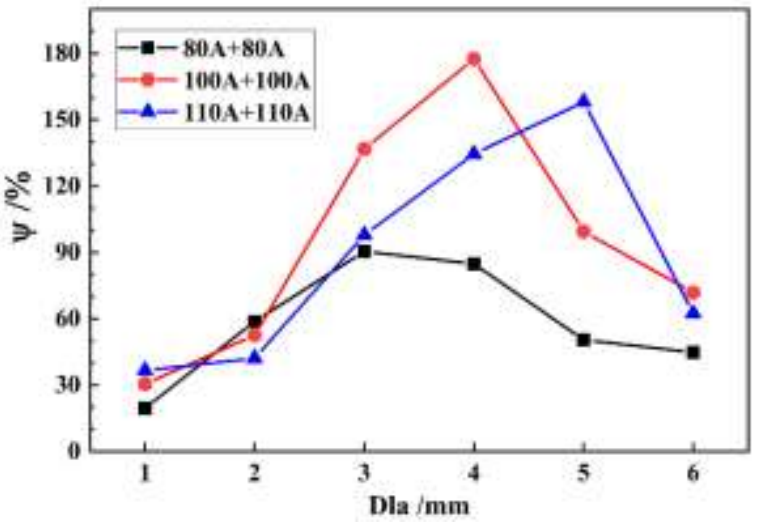

Fig. 4 Change of melting energy increment

\subsection{Plasma and keyhole behavior in hybrid welding}

As mentioned above, the synchronous induction effect of the laser to double-arc greatly improves the energy utilization efficiency of the arc. This was confirmed through monitoring the dynamic behavior of plasma and keyhole in hybrid welding. A high-speed camera and auxiliary light source were used to observe the details of Ar plasma and keyhole in hybrid welding, as shown in Fig. 5. The discharge behavior of double-arc with different Dla was shown in Fig. 6. When Dla is $1 \mathrm{~mm}$, the laser radiates the metal sheet to form a keyhole, the arc plasma expands rapidly $(1 \mathrm{~ms})$ under the impact of the metal vapor in the keyhole, and the discharge space suddenly becomes larger, showing the form of "dispersed plasma". This form is maintained until the keyhole is completely disappeared (4ms). Then the arc returns to its original form. When Dla is $4 \mathrm{~mm}$, the laser acts on the center of molten pool, the liquid high-temperature metal greatly improves the absorption rate of laser energy, the keyhole is then formed quickly. Keyhole plasma attracts the double-arc plasma to move to the vicinity of the keyhole to discharge, and the "coupling plasma" is formed when the laser pulse is applied for $2 \mathrm{~ms}$. In other words, the discharge space of the double-arc plasma shrinks sharply under the induced action of pulsed laser, and the two arcs are coupled to form a bright common conductive channel. And after the laser pulse period is over, although the laser energy is suspended, the coupling discharge still continues for $5 \mathrm{~ms}$. When the keyhole is completely disappeared at $8 \mathrm{~ms}$, the double-arc plasma gradually returns to the original arc form from the coupling form. When Dla is $6 \mathrm{~mm}$, the laser acts on the rear edge of the molten pool. The larger Dla makes the charged particles in keyhole plasma unable to enter the arc to participate in the conduction. There is no induced discharge in arc plasma. Therefore, under this positional relationship, the double-arc plasma always maintains the original arc shape. 


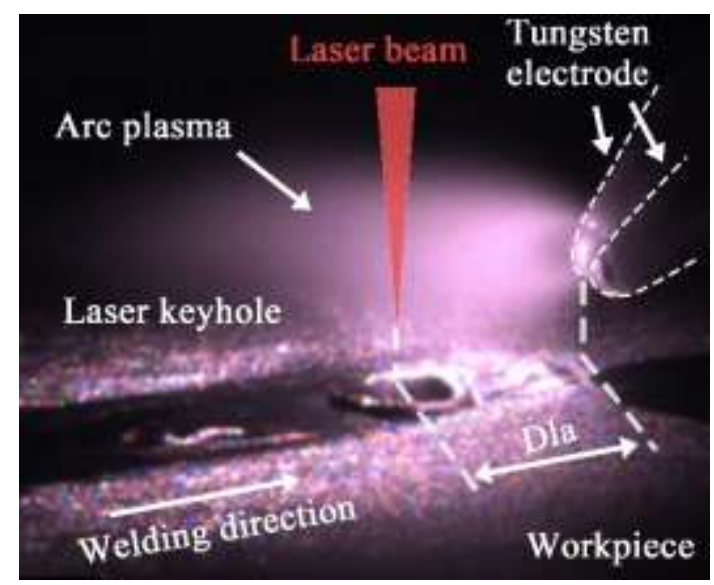

Fig. 5 Details of Ar plasma and keyhole in hybrid welding (Laser pulse energy, $15 \mathrm{~J}$; Pulse duration, $3 \mathrm{~ms}$; Double-arc current, 100A+100A; Dla, $4 \mathrm{~mm}$; welding speed, $800 \mathrm{~mm} / \mathrm{min}$ )

(a)

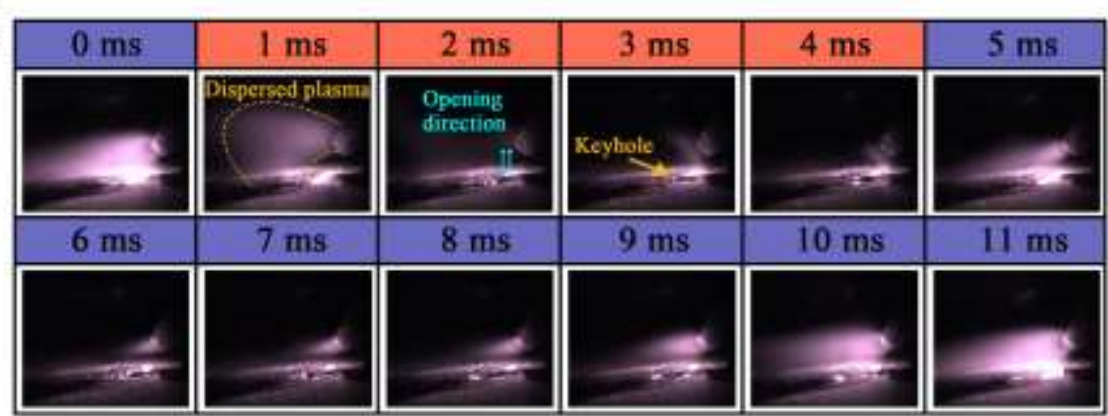

(b)

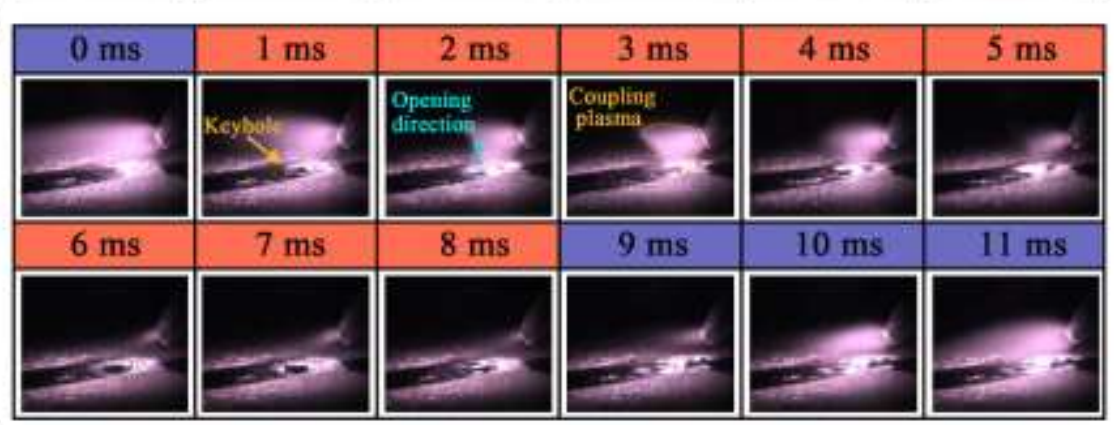

(c)

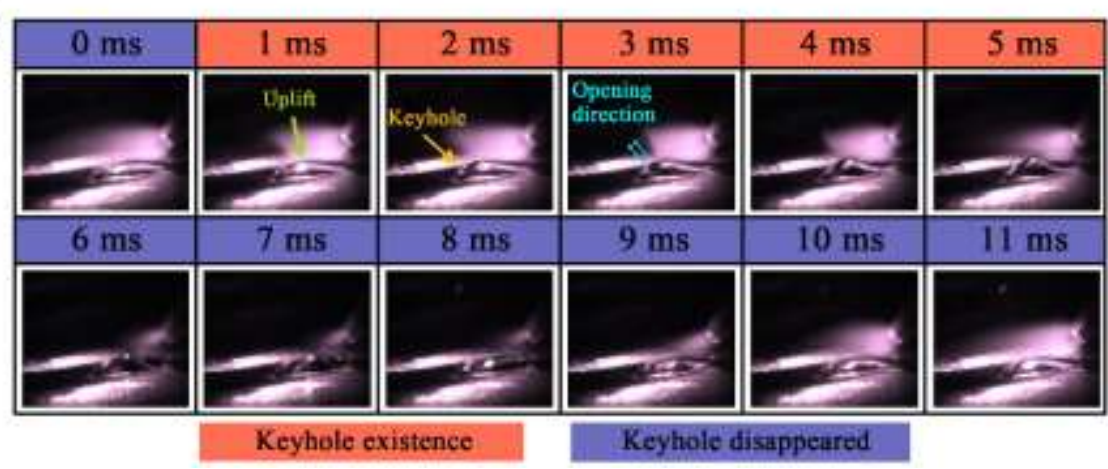

Fig. 6 Discharge behavior of double-arc with different Dla (a) Dla=1 mm (b) Dla=4 mm (c) Dla $=6 \mathrm{~mm}$ (Laser pulse energy, 15 J; Pulse duration, 3 ms; Double-arc current, 100A+100A; welding speed, $800 \mathrm{~mm} / \mathrm{min}$ )

\subsection{Physical characteristics of double-arc plasma}

The spectral radiation information of the plasma can directly express the composition of the charged particles in the arc discharge space. As the intrinsic physical property of plasma, electron temperature can also reflect the 
discharge state of the heat source. The assumptions, calculation methods, check methods for LTE and optical thin of the welding plasma can be found in references [26]. A spectrometer is used to collect the spectral information of the arc center position under different Dla (Fig. 2). The spectral lines of the wavelength range of 513.6-520.2nm and 808.9-813.3nm are shown in Fig. 7. The double-arc plasma and the laser-induced plasma have different interaction states due to different positional relationships. Fig. 7 (a) and (b) respectively show the radiation intensity changes of $\mathrm{Mg}$ atom (Mg I 516.732nm) and Ar atom (Ar I 811.531nm) spectrum in arc plasma. When Dla is $4 \mathrm{~mm}$, the radiation intensity of Mg atom in the plasma rises sharply (Fig. 7 (a)), from 23976 to 51191, while the Ar atom shows the opposite trend (Fig. 7 (b)). Strong coupling discharge occurred between the two kinds of plasma. This is due to the addition of $\mathrm{Mg}$ atoms with lower ionization energy $(7.68 \mathrm{eV})$ in the Ar arc plasma, replacing the Ar atoms $(15.84 \mathrm{eV})$ that are harder to ionize. The change of particle composition leads to a significant drop in the plasma electron temperature (8600K), as shown in Fig. 8. When Dla is 2, 3, and $5 \mathrm{~mm}$, as the positional relationship between the two plasmas changes, the coupling discharge effect becomes weaker. The fluctuation of the radiation intensity of $\mathrm{Mg}$ atoms and $\mathrm{Ar}$ atoms becomes smaller. Compared with the strong coupling discharge, the electron temperature of the weak coupling discharge has risen, but it is lower than that of traditional double-arc (13100K). When Dla is $1 \mathrm{~mm}$, according to the arc behavior in Fig. 6(a), we can assume that the double-arc plasma and the laser-induced plasma have "mutual interference". The abnormal expansion of arc plasma leads to a decrease in the spectral radiation intensity of $\mathrm{Mg}$ atoms and $\mathrm{Ar}$ atoms, and reduces the particle density in the arc space. The lower probability of particle collision increases the speed of electron movement, which ultimately results in a higher electron temperature $(15800 \mathrm{~K})$ than the traditional double-arc (Fig. 8). When the distance between the two plasmas is far (Dla $=6 \mathrm{~mm}$ ), the spectral radiation intensity of plasma is basically unchanged, which is consistent with the behavior of arc plasma in Section 3.2.
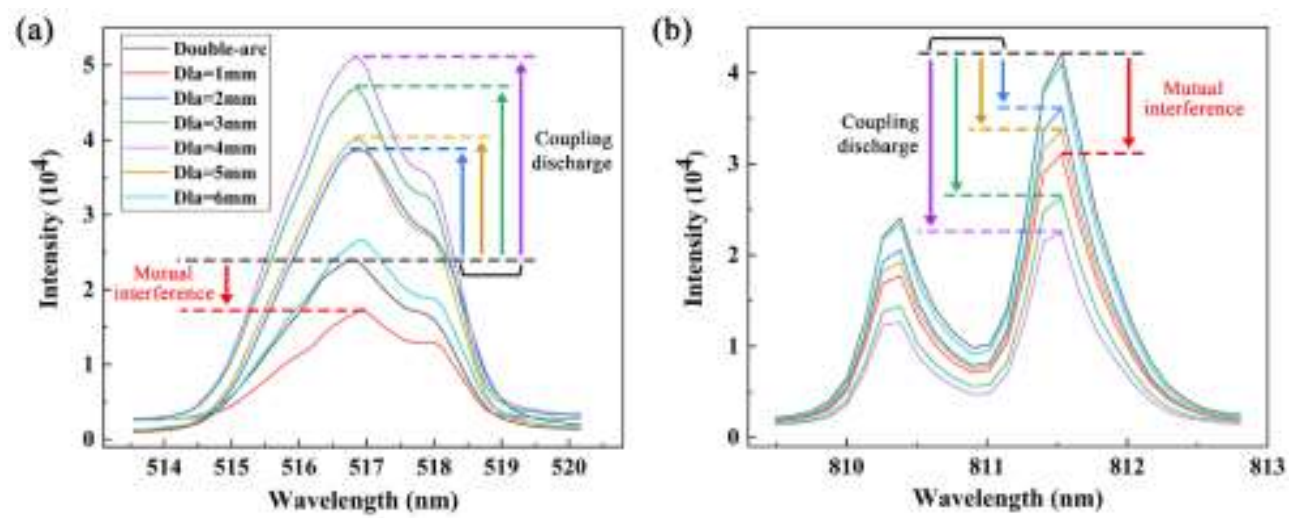

Fig. 7 Spectral radiation intensity of double-arc at different Dla (a) Mg I 516.732nm (b) Ar I 811.531nm (Laser pulse energy, $15 \mathrm{~J}$; Pulse duration, $3 \mathrm{~ms}$; Double-arc current, 100A+100A; welding speed, $800 \mathrm{~mm} / \mathrm{min}$ ) 


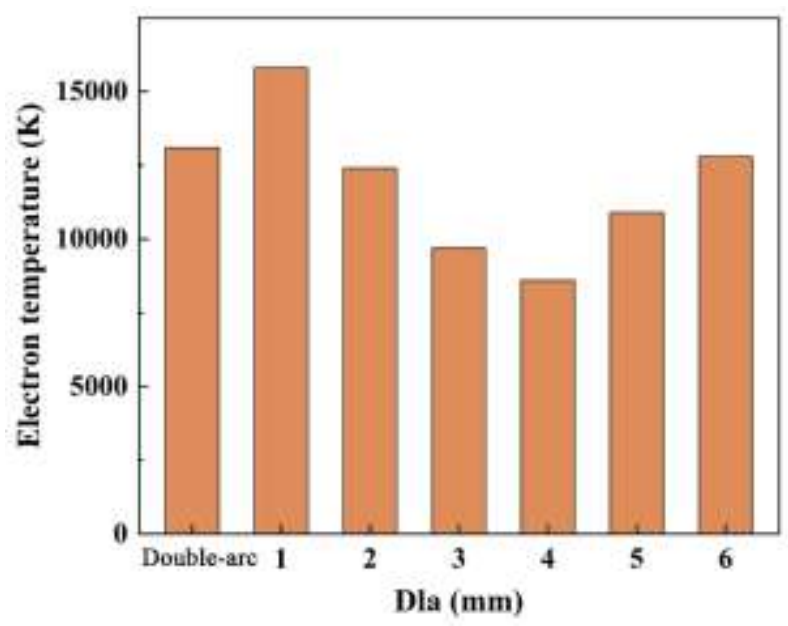

Fig. 8 Electron temperature of double-arc plasma with different Dla

In the hybrid welding process, energy density is considered as a physical quantity that can intuitively characterize the arc characteristics. The above analysis shows that when Dla is $4 \mathrm{~mm}$, the keyhole exists stably, the laser-induced double-arc generates a strong coupling discharge, the coupling double-arc plasma is formed, and the heat source exhibits high energy utilization efficiency. Therefore, the energy density of coupling arc under this parameter combination is characterized. According to reports [34, 35], the spatial current density of arc heat source can be expressed by Formulas 6 and 7:

$$
\begin{gathered}
\rho_{H}=\frac{\zeta U I+P_{\text {laser }}}{S} \\
P_{\text {laser }}=\frac{Q}{w_{\mathrm{p}}}
\end{gathered}
$$

Where $\rho_{H}$ is the average energy density of the conductive area, $\zeta$ is the arc energy contribution rate, $U, I$ are the welding arc voltage and current, respectively, $P_{\text {laser }}$ is the laser pulse peak power, $S$ is the area of heat source, $Q$ is the laser pulse energy, $w_{\mathrm{p}}$ is laser pulse period.

The energy of double-arc was analyzed firstly. The arc current was set to $100 \mathrm{~A}+100 \mathrm{~A}$, and the output voltage of traditional double-arc and coupling double-arc was measured with a Hannover analyzer. The voltage value rose from $13.6 \mathrm{~V}$ to $14.9 \mathrm{~V}$. By calculation, the total arc energy of traditional double-arc and coupling double-arc is $2720 \mathrm{~W}$ and $2980 \mathrm{~W}$, respectively. The arc discharge space has regional characteristics. The welding energy is mainly transferred to the plate through the central conductive area of arc, and the contact surface between the central conductive area and the plate is elliptical. The area and energy contribution rate of the central conductive area were measured and analyzed by the spectrum analyzer. The method for measuring the area of the central conductive area is shown in Fig. 9. Spectral data is collected along the $\mathrm{x}$ and $\mathrm{y}$ axes at a position $0.5 \mathrm{~mm}$ away from the sheet surface. The discharge area of coupling double-arc is smaller than that of traditional double-arc, so the collection step length of traditional double-arc and coupling double-arc is set to $0.5 \mathrm{~mm}$ and $0.2 \mathrm{~mm}$, respectively. The position of the optical fiber probe of spectrometer is adjusted by the spiral micrometer translation stage with an accuracy of 0.003 $\mathrm{mm}$, which can precisely locate the collection position. The Mg I $516.732 \mathrm{~nm}$ spectral line intensity at each position of arc was observed, and the intensity mutation point was determined as the regional boundary point. The result is shown in Fig. 10. The calculated data about the area of central conductive zone in arc can be read from the figure. 
(a)

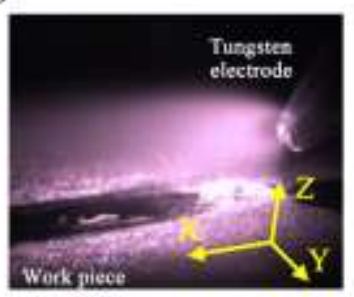

(b)

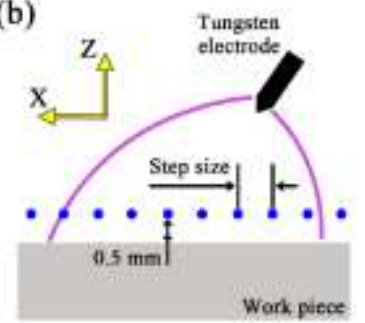

(c)

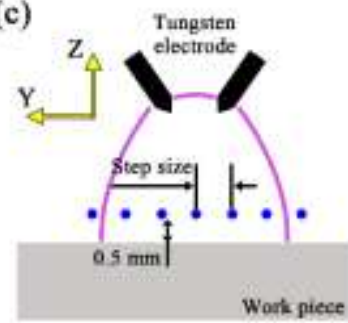

Fig. 9 Measurement method of central conductive area

(a)

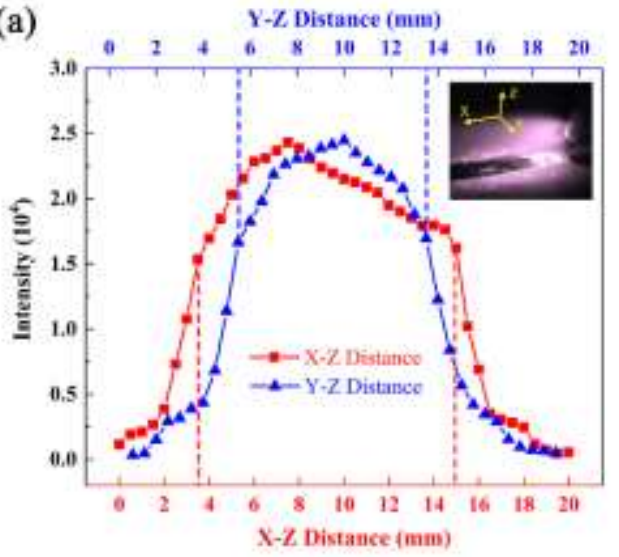

(b)

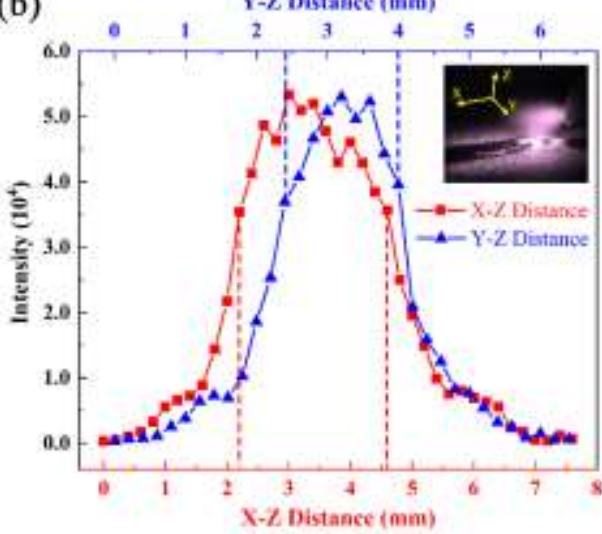

Fig. 10 Spectral intensity of Mg I 516.732nm at various positions of double-arc

(a) Traditional double-arc (b) Coupling double-arc

The conductivity of each area of arc is different, which is related to the current density of each area [36]. It can be seen from Formula 8 that the current density of arc is proportional to the electron density [37].

$$
j=\frac{e^{2} n_{e} \lambda_{e} E}{\sqrt{8 m_{e} k T_{e} / \pi}}
$$

Where $j$ is the current density, $e$ is the electron charge, $n_{e}$ is the electron density at a certain point in the arc, $\lambda_{e}$ is the electron mean free path, $E$ is the electric field strength, $m_{e}$ is the electron mass, $k$ is Boltzmann constant, $T_{e}$ is the electron temperature.

The energy contribution rate can be expressed by the average electron density specific gravity of each arc area, and the calculation method is as follows:

$$
\zeta=\frac{\bar{n}_{\mathrm{ec}}}{\bar{n}_{\mathrm{ec}}+\bar{n}_{\mathrm{eo}}}
$$

Where $\zeta$ is the energy contribution rate of the central conductive region, $\bar{n}_{\mathrm{ec}}$ is the average electron density of the central conductive region, $\bar{n}_{\mathrm{eo}}$ is the average electron density of the outer region of the arc.

The statistical results of the electron density in each arc area are shown in Fig. 11. The average electron density of central conductive area of traditional double-arc is $1.03 \times 10^{17} \mathrm{~cm}^{-3}$, while that of coupling double-arc during pulsed laser induced discharge reaches $1.49 \times 10^{17} \mathrm{~cm}^{-3}$, an increase of $44.7 \%$. The electron density in the outer region of arc is about $10^{15} \sim 10^{16} \mathrm{~cm}^{-3}$. Compared with the central region of arc, the conductivity is significantly reduced. The calculation results of the average energy density are shown in Table 3. It can be seen that the average energy density of hybrid welding is 96 times that of double-arc welding.

The spatial distribution of the energy density of traditional double-arc and coupling double-arc before and after the laser pulse is calculated and imaged fitting, shown in Fig. 12. It can be seen from the figure that, compared with 
traditional double-arc, the coupling double-arc exhibits a steeper energy gradient and a higher energy density. The maximal energy density of double-arc increased 9.3 times from $4.15 \times 10^{3} \mathrm{~W} / \mathrm{cm}^{2}$ to $3.87 \times 10^{4} \mathrm{~W} / \mathrm{cm}^{2}$. With laser action, the energy density of the entire central conductive area is above $2.21 \times 10^{4} \mathrm{~W} / \mathrm{cm}^{2}$, much higher than $1.07 \times 10^{3} \mathrm{~W} / \mathrm{cm}^{2}$ without laser action. The increase of energy density is mainly due to the coupling-enhanced effect between arcs under laser induction.

(a)

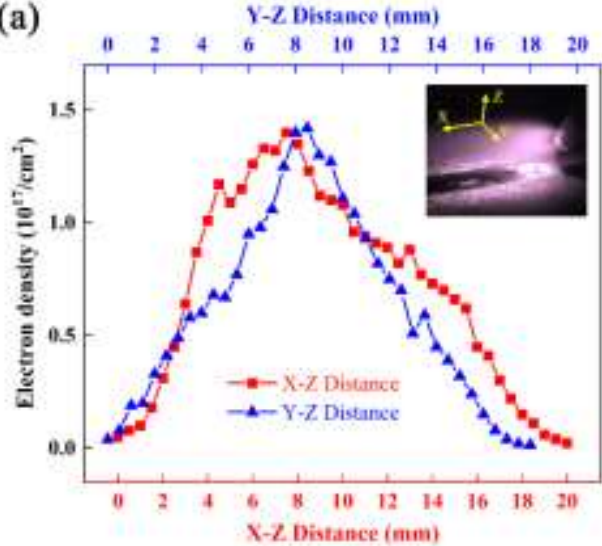

(b)

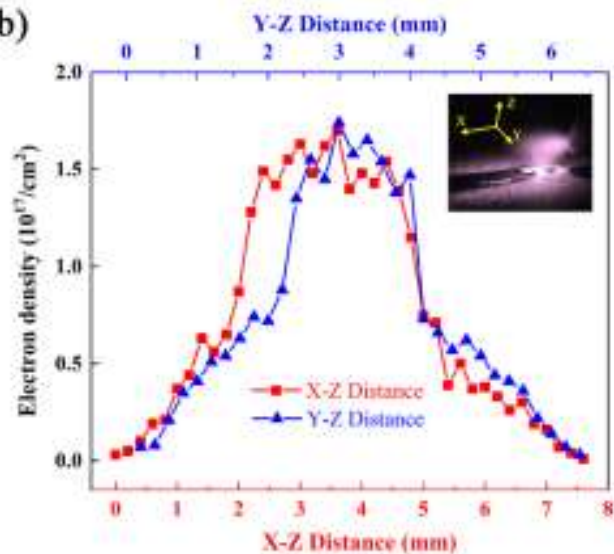

Fig. 11 Electron density at various positions of arc (a) Traditional double-arc (b) Coupling double-arc

Table 3. Calculation results of average energy density of hybrid heat source.

\begin{tabular}{|c|c|c|c|c|c|c|c|}
\hline Welding process & $\zeta$ & $U I / \mathrm{W}$ & $X Z / \mathrm{mm}$ & $Y Z / \mathrm{mm}$ & $S / \mathrm{mm}^{2}$ & $P_{\text {laser }} / \mathrm{W}$ & $\rho_{H} / \mathrm{W} \cdot \mathrm{cm}^{-2}$ \\
\hline Double-TIG & $66.73 \%$ & 2720 & 11.5 & 8 & 72.22 & - & $2.51 \times 10^{3}$ \\
\hline Hybrid welding & $74.78 \%$ & 2980 & 2.4 & 1.6 & 3.014 & 5000 & $2.4 \times 10^{5}$ \\
\hline
\end{tabular}

(a)

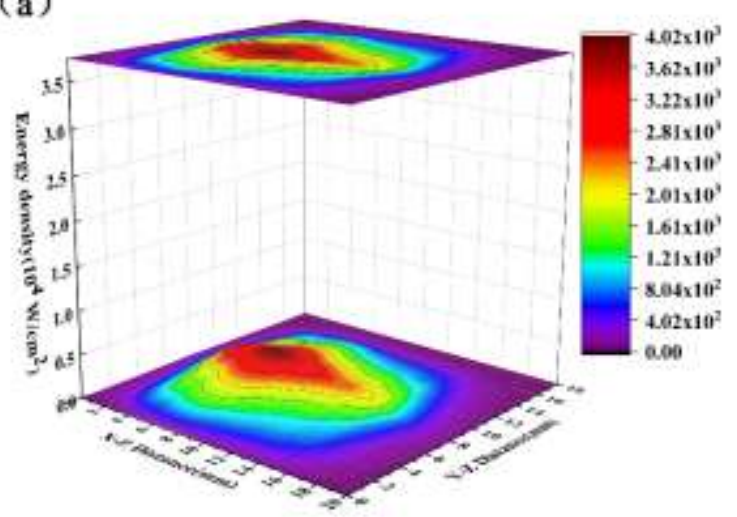

(b)

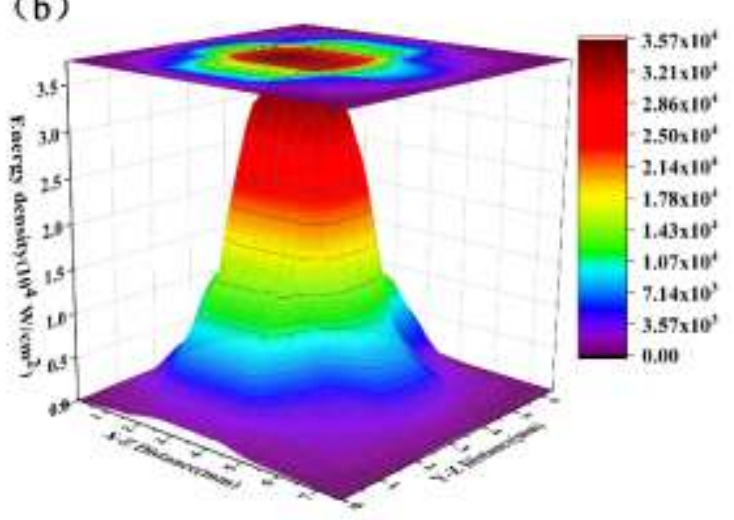

Fig. 12 Spatial distribution of energy density of double-arc (a) Traditional double-arc (b) Coupling double-arc

\section{Discussion}

\subsection{Particle migration of Laser-induced double-arc}

The above quantitative analysis shows that the induced effect of laser to double-arc plasma greatly increases the energy density of arc. The increase in energy density indicates that the heat source has a stronger heating capacity for the workpiece. The particle migration process of laser-induced double-arc coupling discharge is analyzed. The charged particles in arc plasma usually consist of free electrons, metal ions and shielding gas ions. In this experiment, the cations are mainly Mg ions and Ar ions. In the double-arc free discharge stage, when the ionization and 
recombination of atoms in plasma reach the ideal state of dynamic equilibrium, the charged particles in the arc column are uniformly distributed in the arc discharge space. However, according to the plasma physics theory, there is a difference in particle concentration in the radial direction of arc column area due to the diffusion effect. The edge of the arc column has a higher electron concentration, and the center area has a higher ion concentration, as shown in Fig. 13(a). The position where electrons are injected into the anode changes continuously with the state of the anode surface. The electrons tend to move in the direction with the largest potential gradient, and finally flow into the anode where the barrier is relatively low. When the laser pulse is applied, a new highly discharged plasma (keyhole plasma) replaces the anode spots on the surface of the sheet as a channel for electrons to enter the anode. The potential of keyhole plasma is almost the same as that of the anode, while the resistivity is much lower. The direction of electric field changes from "tungsten electrode-workpiece" to "tungsten electrode-keyhole plasmaworkpiece". In welding process, the current flows from the workpiece to the tungsten electrode, forming an electric field directed to the tungsten electrode, and the arc induces a magnetic field perpendicular to the paper surface in the space. The charged particles in the plasma move under the drive of electromagnetic field. The characteristics of arc plasma are closely related to the movement and migration of charged particles. Before and after the laser is applied, the force state of the charged particles in the arc1 space is shown in Fig. 14. According to the left-hand rule, the cation receives the electric field force and the Lorentz force directed to the arc2 column, while the electron receives the opposite electric field force to the cation. The force on charged particle can be expressed by Equation 10 :

$$
\vec{F}=\vec{F}_{\mathrm{E}}+\vec{F}_{\mathrm{B}}=\mathrm{q} \overrightarrow{\mathrm{E}}+\mathrm{q} \overrightarrow{\mathrm{v}} \times \overrightarrow{\mathrm{B}}
$$

Where $\vec{F}$ is the total force on the charged particles, $\vec{F}_{\mathrm{E}}$ is the electric field force, $\vec{F}_{\mathrm{B}}$ is the Lorentz force, $\mathrm{q}$ is the electric charge, $\vec{E}$ is the electric field strength, $\vec{v}$ is the speed of motion, $\vec{B}$ is the magnetic field strength.

Regardless of external environmental factors, the charged particles move and migrate in the direction of the force, and finally become stable, as shown in Fig. 13. It can be seen from Equation 11 that the thermal motion speed of charged particles is inversely proportional to the particle mass [38].

$$
\mathrm{v}=\sqrt{\mathrm{kT} / \mathrm{m}}
$$

Where $\mathrm{k}$ is Boltzmann constant, $\mathrm{T}$ is the particle temperature, $\mathrm{m}$ is the particle mass.

Electrons move faster than cations because of their smaller mass. In the initial stage of laser action, the electrons in the double-arc discharge space quickly gather near the keyhole and exchange energy with the keyhole plasma, as shown in Fig. 13(b). The cations move towards the tungsten electrode driven by the electromagnetic field, and the double-arc discharge space shrinks, as shown in Fig. 13(c). Finally, as a large number of Mg particles in keyhole plasma continue to migrate into the arc column, instead of Ar particles with higher ionization energy (15.84 eV) to participate in the conduction. A compressed conductive channel shared by the two arcs is formed, and the coupling discharge tends to be stable, as shown in Fig. 13 (d). The contraction of the double-arc conduction channel greatly increases the electron density of the plasma and promotes the energy transfer between the double-arc and the metal sheet.

For DC positive arc discharge, the keyhole plasma provides an anode inlet with high conductivity for electrons, which exhibits an electron collection effect. In AC mode, the charged particles in the keyhole plasma migrate between the cathode and the anode over time. When the keyhole plasma is used as the cathode, electrons are more easily ejected from the plasma than from the plate since there is no work function. The collection and emission of charged particles by the keyhole plasma can induce the double-arc plasma to discharge into the keyhole, compress the conductive channel of the double-arc, and increase its energy density. 
(a)

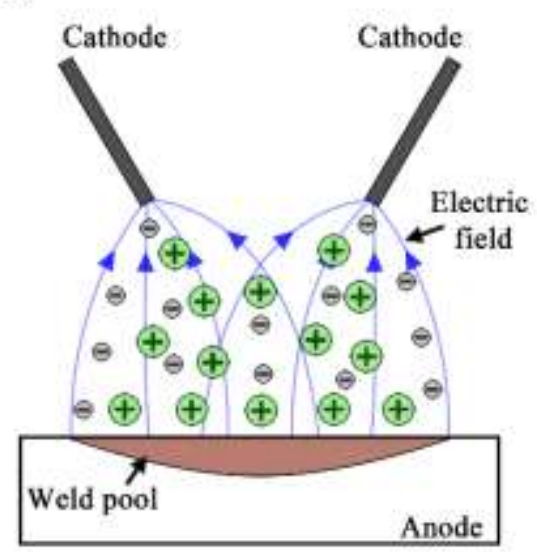

(c)

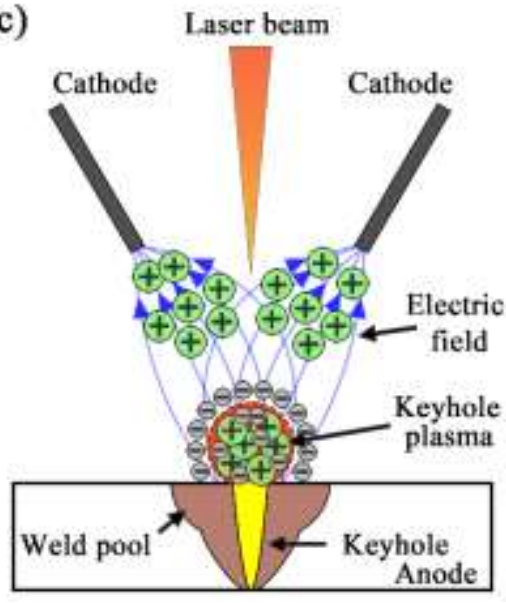

(b)

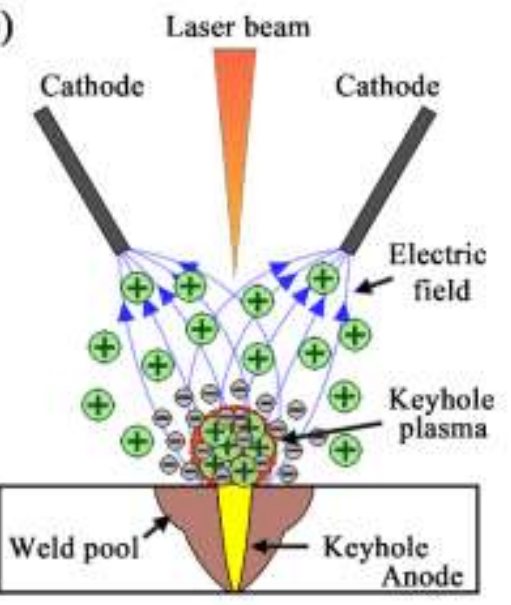

(d)

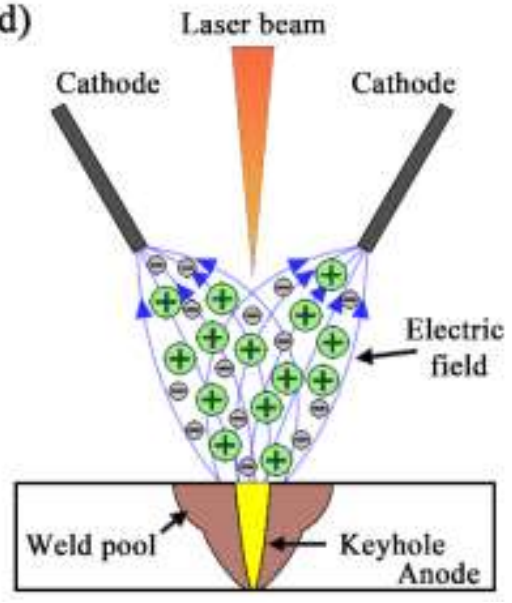

Fig. 13 Schematic diagram of particle migration in double-arc induced by laser

(a) Double-arc free discharge stage (b) Laser-induced initial stage

(c) Laser-induced contraction stage (d) Laser-induced stable stage
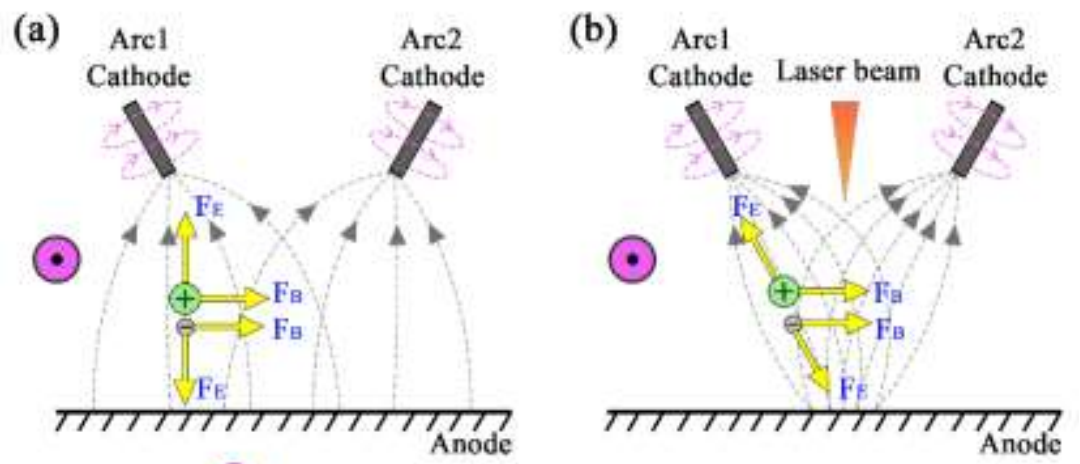

Magnetic field direction produced by Are 2

Fig. 14 Schematic diagram of particle force (a) Traditional double-arc (b) Coupling double-arc

\subsection{Electron kinetic energy of plasma}

During the energy transport process of arc plasma, the electrons and ions in the arc column move in the direction of electric field, converting the lost potential energy into kinetic energy. However, due to the difference in mass, the speed of electrons is much greater than that of cations. Therefore, the role of energy transport is almost entirely borne by electrons [37]. It can be said that the physical state of electrons has a significant impact on the 
energy transport process of the arc.

In the plasma discharge space, electrons are in an irregular motion state after colliding with other charged particles, so the average value of their velocity in the direction of electric field is zero. The mass of electron is represented by $m_{\mathrm{e}}$, the electric quantity of the electron is represented by $e$, the mean free path of the electron is represented by $\lambda$, and the average speed of the electron is represented by $\vec{C}$. Then the average movement time of the electron in two adjacent collisions is $\tau=\frac{\lambda}{\vec{C}}$, and at this time the electron obtains the acceleration $e X / m_{\mathrm{e}}$ from the electric field with the field strength X. Since the average velocity of electrons in the direction of the electric field is zero, the average movement distance of electrons in the direction of the electric field can be expressed as:

$$
l=\frac{1}{2} \frac{e X \cdot \tau^{2}}{m_{\mathrm{e}}}
$$

The average velocity $u_{\mathrm{e}}$ of electrons in the direction of the electric field can be expressed as:

$$
u_{\mathrm{e}}=\frac{l}{\tau}=\frac{1}{2} \frac{e X \cdot \tau}{m_{\mathrm{e}}}=\frac{1}{2} \frac{e \lambda}{m_{\mathrm{e}} \vec{C}} \cdot X
$$

However, the free path of electrons varies in different states. Considering the distribution of the free path of electrons, $u_{\mathrm{e}}$ can be expressed as:

$$
\begin{gathered}
u_{\mathrm{e}}=\frac{e \lambda}{m_{\mathrm{e}} \vec{C}} \cdot X=K_{\mathrm{e}} X \\
K_{\mathrm{e}}=\frac{e \lambda}{m_{\mathrm{e}} \vec{C}}
\end{gathered}
$$

Where $K_{e}$ represents the mobility of electrons.

In addition, the average electron velocity $\vec{C}$ has the following relationship with the electron temperature $T_{\mathrm{e}}$, where $M$ is the molar mass:

$$
\vec{C}=\sqrt{\frac{8 k T_{\mathrm{e}}}{\pi M}}=1.45 \times 10^{4} \cdot \sqrt{\frac{T_{\mathrm{e}}}{M}}
$$

The mean free path $\lambda$ of electron can be expressed as:

$$
\lambda=\frac{1}{n_{\mathrm{e}} \pi \gamma^{2}}
$$

Where $n_{\mathrm{e}}$ is the electron density, $\gamma$ is the electron radius. Combining the above formula, the total kinetic energy $E$ of the electron can be expressed as:

$$
E=\frac{1}{2} n_{\mathrm{e}} m_{\mathrm{e}} u_{\mathrm{e}}^{2}=4 \times 10^{18} \frac{m_{\mathrm{e}} X^{2}}{n_{\mathrm{e}} T_{\mathrm{e}} \pi^{2} \gamma^{4}}
$$

Formula 18 is simplified as follows:

$$
E \propto \frac{X^{2}}{n_{\mathrm{e}} T_{\mathrm{e}}}
$$

Obviously, the total kinetic energy of electrons $E$ is proportional to $X^{2}$ and inversely proportional to $n_{\mathrm{e}}$ and $T_{\mathrm{e}}$. By means of spectral diagnosis, it is calculated that the electron temperature at the center of the arc (Fig. 2) decreased from $13100 \mathrm{~K}$ to $8600 \mathrm{~K}$, and the electron density at the same point increased from $1.35 \times 10^{17} \mathrm{~cm}^{-3}$ to $1.79 \times 10^{17} \mathrm{~cm}^{-3}$. After monitoring the arc voltage, it is found that the electric field intensity has increased from 68 $\mathrm{V} / \mathrm{cm}$ to $74.5 \mathrm{~V} / \mathrm{cm}$. Putting the data results into the above formula, it can be found that the total kinetic energy of electrons of coupling double-arc is increased by $38 \%$ compared with traditional double-arc, which promotes the rapid transfer of arc energy. This indicates that the induction effect of keyhole plasma generated by the high-energy laser on the double-arc plasma promotes the coupling discharge between the arcs and changes the motion state of the electrons in the discharge space, thereby increasing the energy density and the energy utilization efficiency of 
the double-arc.

\section{Conclusion}

This paper quantitatively calculates the energy utilization efficiency of pulsed laser induced double-arc. The physical characteristics of double-arc are studied by the spectral diagnosis method, and a physical model is established to analyze the particle migration process during coupling discharge, and to characterize the electronic movement state. Conclusions are as below:

(1) During laser-double arc hybrid welding, Dla has a significant impact on the energy utilization efficiency of heat source. As Dla becomes larger, there are three interactions between double-arc plasma and keyhole plasma: Mutual interference, Coupling-enhanced discharge, and No interaction. As the double-arc current increases with other welding parameters unchanged, the Dla value for the joint to obtain the highest melting energy increment also increases. When the arc current is $100 \mathrm{~A}+100 \mathrm{~A}$ and Dla is $4 \mathrm{~mm}$, a strong coupling discharge occurs, and the melting energy increment $\psi$ reaches $177.5 \%$.

(2) The synchronous induction of laser to double-arc plasma promotes the coupling-enhanced discharge between the arcs, which greatly increases the energy density of double-arc. The average energy density of hybrid welding is 96 times that of double-arc welding. In addition, under the induced effect of laser pulse, the energy density of double-arc increases 9.3 times from $4.15 \times 10^{3} \mathrm{~W} / \mathrm{cm}^{2}$ to $3.87 \times 10^{4} \mathrm{~W} / \mathrm{cm}^{2}$. This greatly increases the heating capacity of double-arc.

(3) The particle migration when the coupling discharge occurs is divided into four stages: Double-arc free discharge stage, Laser-induced initial stage, Laser-induced contraction stage and Laser-induced stable stage. The Mg particles in keyhole plasma migrate into the arc column, which promotes the formation of the compressed conductive channel shared by the double-arc. The total kinetic energy of electrons of coupling double-arc increases by $38 \%$ compared with traditional double-arc.

\section{Declarations:}

a. Funding: This work was supported by the National Key R\&D Program of China (2018YFB1107902) organization name: Ministry of Science and Technology of the People's Republic of China.

b. Conflicts of interest/Competing interests: The authors declare that they have no known competing financial interests or personal relationships.

c. Availability of data and material: All the data have been presented in the manuscript.

d. Code availability: (Not applicable).

e. Ethics approval: The paper follows the guidelines of the Committee on Publication Ethics (COPE).

f. Consent to participate: The authors declare that they all consent to participate this research.

g. Consent for publication: The authors declare that they all consent to publish the manuscript.

h. Authors' contributions

Liming Liu（刘黎明）: Conceptualization; Funding acquisition;

Xinkun Xu（徐信坤）: Roles/Writing - original draft; Methodology; Formal analysis;

Guomin Xu（徐国敏）: Data curation; Supervision;

Zhaodong Zhang （张兆栋）: Writing - review \& editing; Validation;

\section{References:}

[1] Muñoz J A, Komissarov A, Mejía I, Hernández-Belmontes H, Cabrera J-M (2021) Characterization of the Gas Tungsten Arc Welding (GTAW) joint of Armco iron nanostructured by Equal-Channel Angular Pressing (ECAP). J Mater Process Tech 288. 
[2] Singh A, Singh R P (2020) A review of effect of welding parameters on the mechanical properties of weld in submerged arc welding process. Mater Today: Proceedings 26: 1714-1717.

[3] Balasubramanian T S, Balakrishnan M, Balasubramanian V, Manickam M A M (2011) Influence of welding processes on microstructure, tensile and impact properties of Ti-6Al-4V alloy joints. T Nonferr Metal Soc 21(6): 1253-1262.

[4] Balasubramanian V, Jayabalan V, Balasubramanian M (2008) Effect of current pulsing on tensile properties of titanium alloy. Mater Design 29(7): 1459-1466.

[5] Cheng Z, Ye Z, Huang J, Yang J, Chen S, Zhao X (2020) Influence of heat input on the intermetallic compound characteristics and fracture mechanisms of titanium-stainless steel MIG-TIG double-sided arc welding joints. Intermetallics 127.

[6] Wu K, Cao X, Yin T, Zeng M, Liang Z (2019) Metal transfer process and properties of double-wire double pulsed gas metal arc welding. J Manuf Process 44: 367-375.

[7] Ma Z, Zhuang M, Li M (2020) Effect of main arc voltage on arc behavior and droplet transfer in tri-arc twin wire welding. J Mater Res Technol 9(3): 4876-4883.

[8] Chen J, Lu Y, Li X, Zhang Y (2012) An innovative GTAW process attains high-speed filler metal melting to compete with GMAW in deposition. Weld J 91: 261-269.

[9] Reis R P, Scotti A, Norrish J, Cuiuri D (2013) Investigation on welding arc interruptions in the presence of magnetic fields: arc length, torch angle and current pulsing frequency influence. IEEE T Plasma Sci 41(1): 133-139. [10] Schwedersky M B, Gonçalves e Silva R H, Dutra J C, Reisgen U, Willms K (2018) Arc characteristic evaluation of the double-electrode GTAW process using high current values. Int J Adv Manuf Tech 98(1-4): 929-936.

[11] Qin G, Meng X, Fu B (2015) High speed tandem gas tungsten arc welding process of thin stainless steel plate. J Mater Process Tech 220: 58-64.

[12] Liu G, Xiong J, Tang L (2020) Microstructure and mechanical properties of 2219 aluminum alloy fabricated by double-electrode gas metal arc additive manufacturing. Addit Manuf 35.

[13] Scalet Rossini L F, Valenzuela Reyes R A, Spinelli J E (2019) Double-wire tandem GMAW welding process of HSLA50 steel. J Manuf Process 45: 227-233.

[14] Zhang G J, Leng X S, Wu L (2006) Physics characteristic of coupling arc of twin-tungsten TIG welding. T Nonferr Metal Soc 16(4): 813-817.

[15] Lu R, Wei H, Li F, Zhang Z, Liang Z, Li B (2020) In-situ monitoring of the penetration status of keyhole laser welding by using a support vector machine with interaction time conditioned keyhole behaviors. Opt Laser Eng 130.

[16] Xu L, Tang X, Zhang R, Lu F, Cui H (2021) Weld bead characteristics for full-penetration laser welding of aluminum alloy under electromagnetic field support. J Mater Process Tech 288.

[17] Hess A, Weber R, Heider A, Graf T (2010) Forced deep-penetration welding with low-power second-harmonic assistance of cw copper welding with $1 \mu \mathrm{m}$ wavelength. Phys Procedia 5: 29-36.

[18] Steen W (1980) Arc augmented laser processing of materials. J Appl Phys 51(11): 5636-5641.

[19] Meng Y, Gao M, Zeng X (2018) Quantitative analysis of synergic effects during laser-arc hybrid welding of AZ31 magnesium alloy. Opt Laser Eng 111: 183-192.

[20] Ragavendran M, Chandrasekhar N, Ravikumar R, Saxena R, Vasudevan M, Bhaduri A K (2017) Optimization of hybrid laser-TIG welding of 316LN steel using response surface methodology (RSM). Opt Laser Eng 94: 27-36.

[21] Zhu Z, Wang W, Li Y, Chen H (2019) Effect of laser-arc offset and laser-deviation angle on the control of a TiAl interlayer. J Mater Process Tech 271: 336-345.

[22] Casalino G, Mortello M, Leo P, Benyounis K Y, Olabi A G (2014) Study on arc and laser powers in the hybrid welding of AA5754 Al-alloy. Mater Design 61: 191-198.

[23] Shinn B W, Farson D F, Denney P E (2013) Laser stabilisation of arc cathode spots in titanium welding. Sci 
Technol Weld Joi 10(4): 475-481.

[24] Hu B, Ouden G (2005) Laser induced stabilisation of the welding arc. Sci Technol Weld Joi 10: 76-81.

[25] Li Z, Wang W, Wang X, Li H (2010) A study of the radiation of a Nd:YAG laser-MIG hybrid plasma. Opt Laser Technol 42(1): 132-140.

[26] Liu L, Xu X, Zhu Y (2020) Study on synchronous induction of arc plasma by laser in laser-double TIG hybrid welding. Opt Laser Eng 133.

[27] Chen M, Xin L, Zhou Q, He L, Wu F (2018) Effect of laser pulse on alternative current arc discharge during laser-arc hybrid welding of magnesium alloy. Opt Laser Eng 100: 208-215.

[28] Griem H (1964) Plasma Spectroscopy. McGraw-Hill, New York.

[29] Hoffman J, Szyma ski Z (2004) Time-dependent spectroscopy of plasma plume under laser welding conditions. J Phys D Appl Phys 37(13): 1792-1799.

[30] Liu L, Chen M (2011) Interactions between laser and arc plasma during laser-arc hybrid welding of magnesium alloy. Opt Laser Eng 49(9-10): 1224-1231.

[31] (2019) National Institute of Standards and Technology database. https://www.nist.gov/pml/atomic-spectradatabase Accessed 25 December 2019.

[32] Mazar Atabaki M, Nikodinovski M, Chenier P, Ma J, Liu W, Kovacevic R (2014) Experimental and numerical investigations of hybrid laser arc welding of aluminum alloys in the thick T-joint configuration. Opt Laser Technol 59: 68-92.

[33] Ready J (1978) Industrial applications of lasers. Academic Press, New York.

[34] Guo Z, Yin S, Qian Z, Liao H, Gu S (2015) Effect of the deviation of the current density profile center on the three-dimensional non-transferred arc plasma torch. Comput Fluids 114: 163-171.

[35] Liu L M, Shi J P, Hou Z L, Song G (2018) Effect of distance between the heat sources on the molten pool stability and burn-through during the pulse laser-GTA hybrid welding process. J Manuf Process 34: 697-705.

[36] Jiang Q X, Zou Y R, Du D (2016) Measurement of current density distribution in welding arc space based on image analysis. Trans China Weld Institution 37(8): 101-104.

[37] AnTeng H, ChangGu C (1985) Welding arc phenomenon. Machinery Industry Press.

[38] Meng Y, Gao M, Zeng X (2018) Effects of arc types on the laser-arc synergic effects of hybrid welding. Opt Express 26(11): 14775-14785. 
Figures

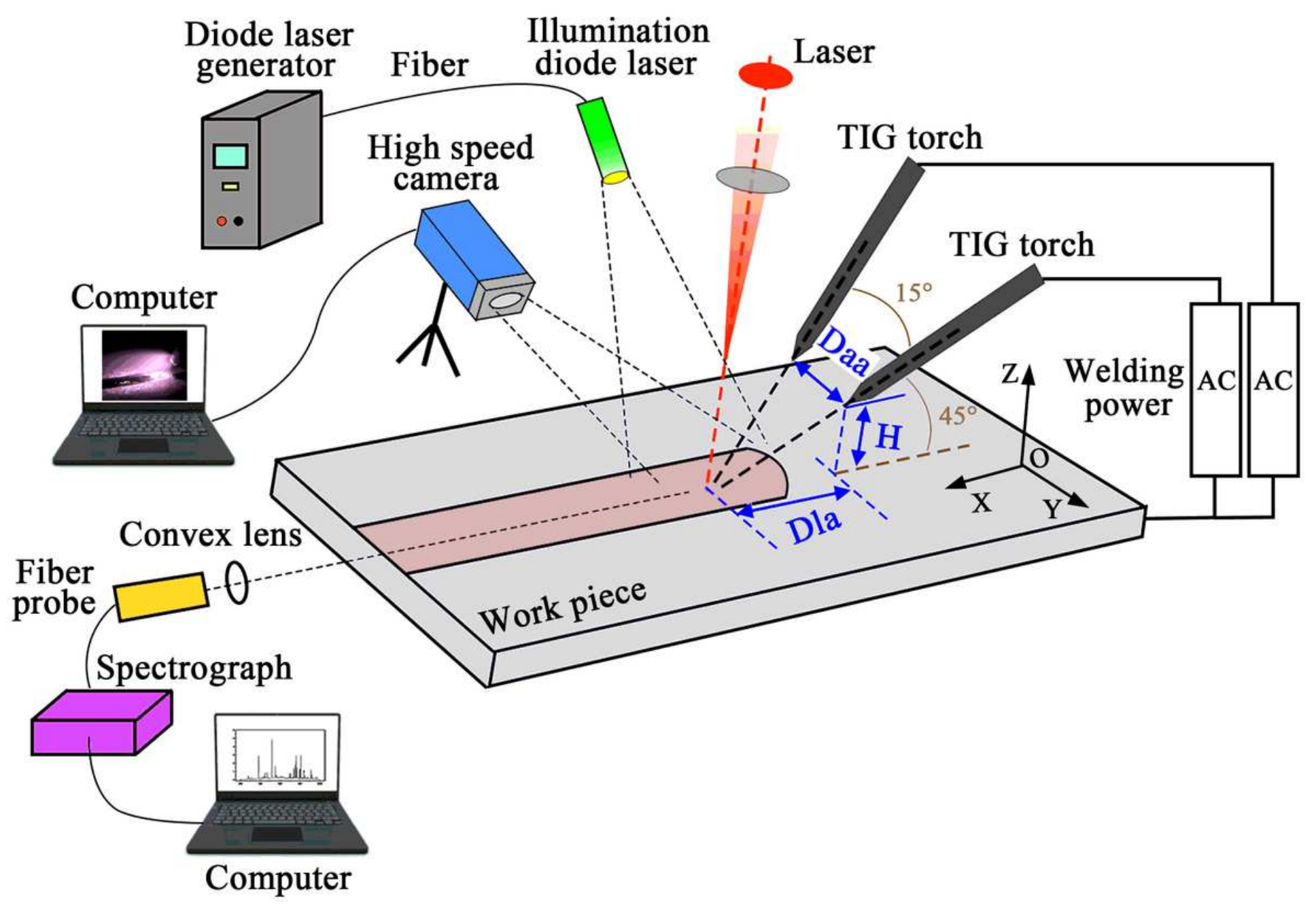

Figure 1

Laser-double TIG hybrid welding and diagnosis system 


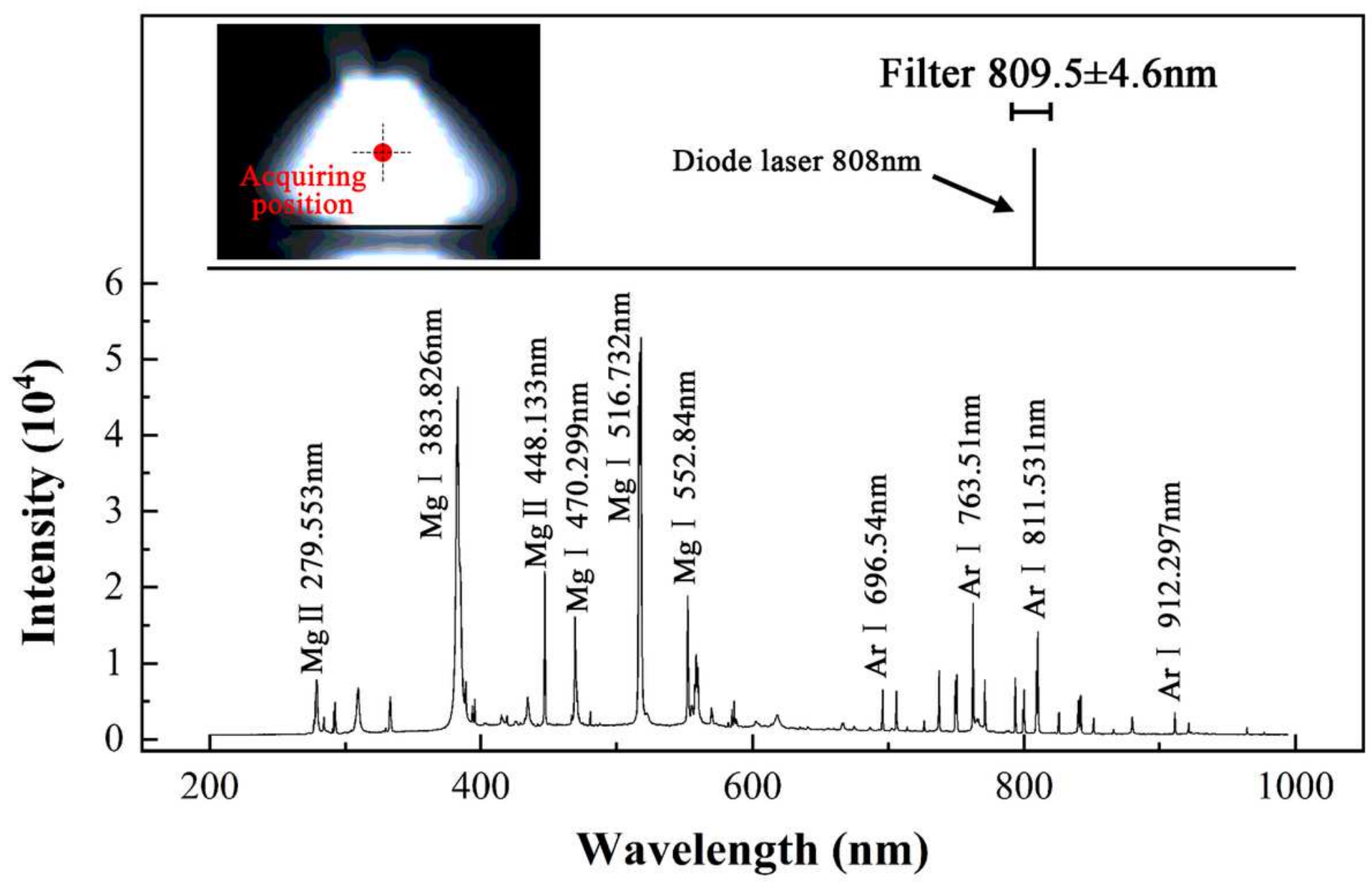

Figure 2

Hybrid welding spectral lines and optical filter

\begin{tabular}{|c|c|c|c|c|c|c|c|c|}
\hline \multirow{2}{*}{$\begin{array}{c}\text { Double-arc } \\
\text { current }\end{array}$} & \multicolumn{6}{|c|}{$\mathrm{Dla} / \mathrm{mm}$} & \multirow{2}{*}{ double-arc } & \multirow{2}{*}{ laser } \\
\cline { 2 - 9 } \\
\hline $80 \mathrm{~A}+80 \mathrm{~A}$
\end{tabular}

Figure 3

Joint morphology under different welding processes 


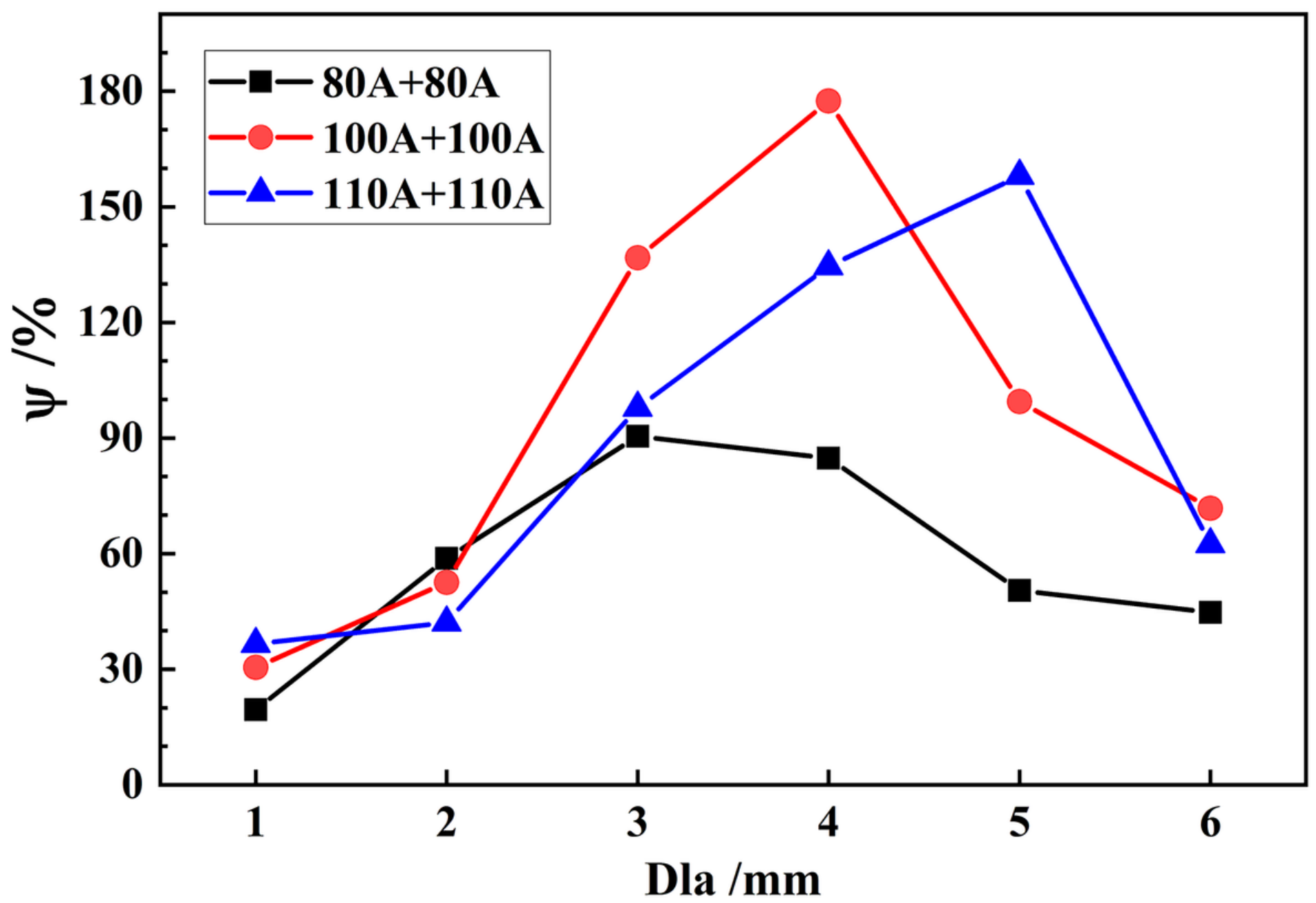

Figure 4

Change of melting energy increment 


\section{Laser beam}

\section{Tungsten electrode,}

\section{Arc plasma}

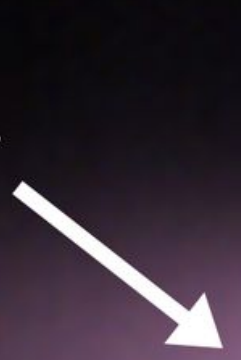

Laser keyhole

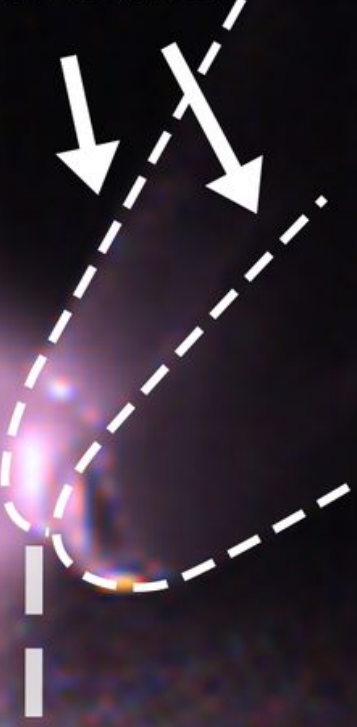

\section{Welding direction}

Workpiece

Figure 5

Details of Ar plasma and keyhole in hybrid welding $ه$ Laser pulse energy, $15 \mathrm{~J}$; Pulse duration, $3 \mathrm{~ms}$; Double-arc current, 100A+100A; Dla, 4 mm; welding speed, 800 mm/min $\llbracket$ 
(a)

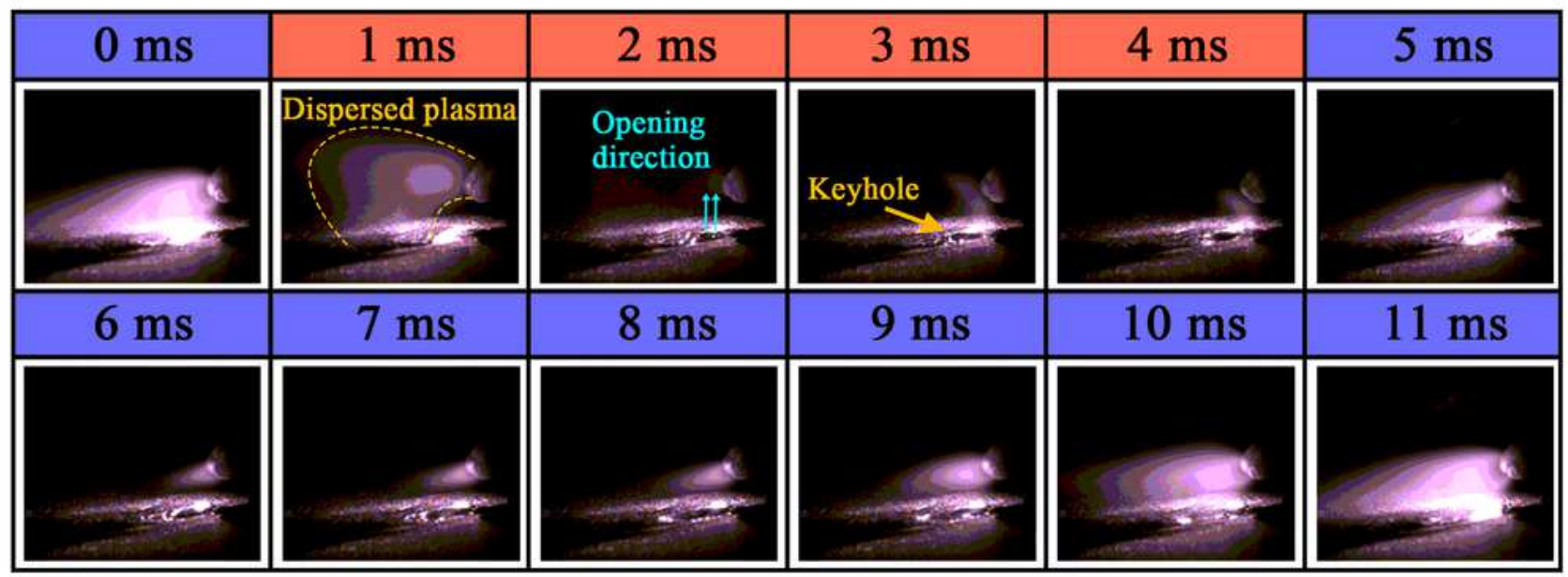

(b)

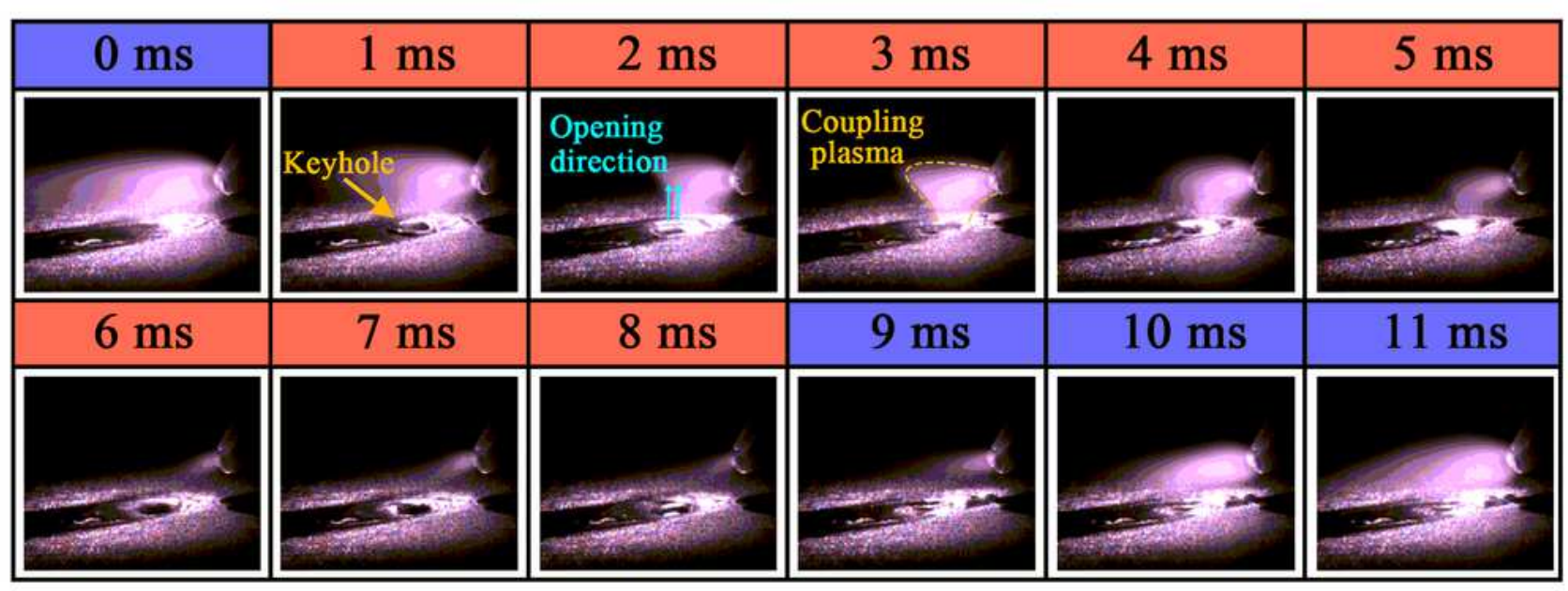

(c)

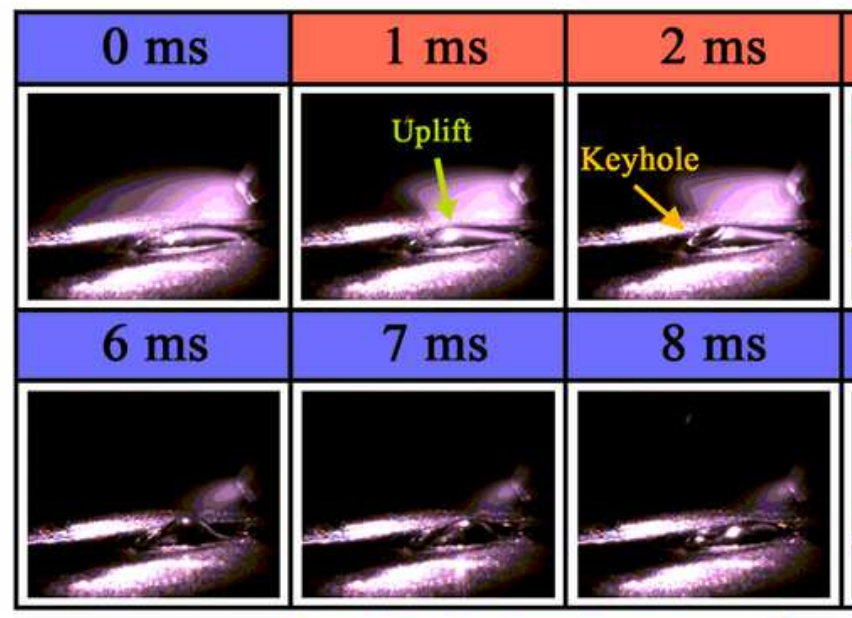

Keyhole existence

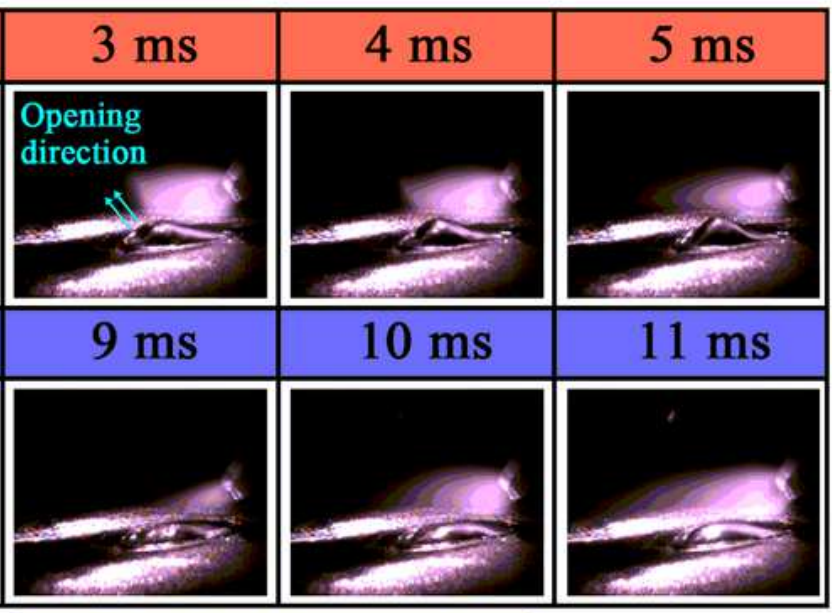

Keyhole disappeared

Figure 6

Discharge behavior of double-arc with different Dla (a) Dla=1 mm (b) Dla=4 mm (c) Dla=6 mm खLaser pulse energy, $15 \mathrm{~J}$; Pulse duration, $3 \mathrm{~ms}$; Double-arc current, 100A+100A; welding speed, $800 \mathrm{~mm} / \mathrm{min} \rrbracket$ 

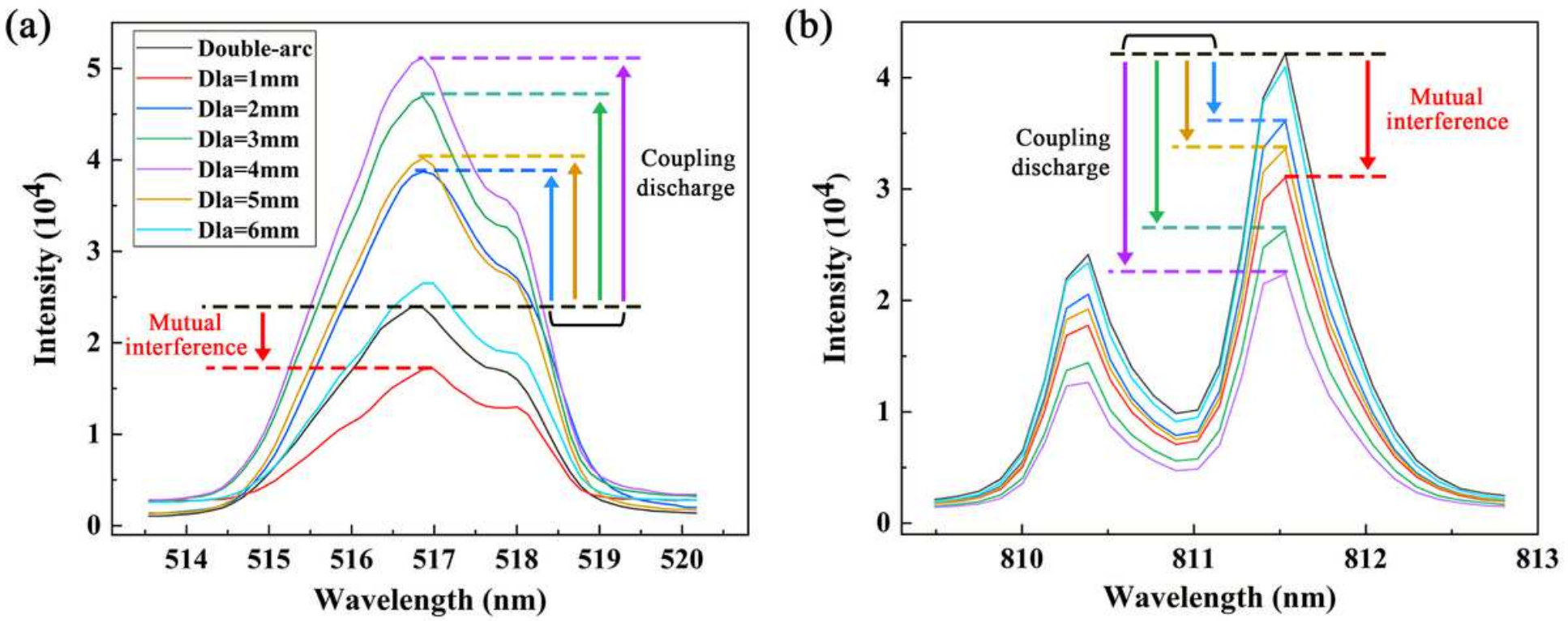

Figure 7

Spectral radiation intensity of double-arc at different Dla (a) Mg I 516.732nm (b) Ar I 811.531nm 囚Laser pulse energy, $15 \mathrm{~J}$; Pulse duration, $3 \mathrm{~ms}$; Double-arc current, 100A+100A; welding speed, $800 \mathrm{~mm} / \mathrm{min} \rrbracket$ 


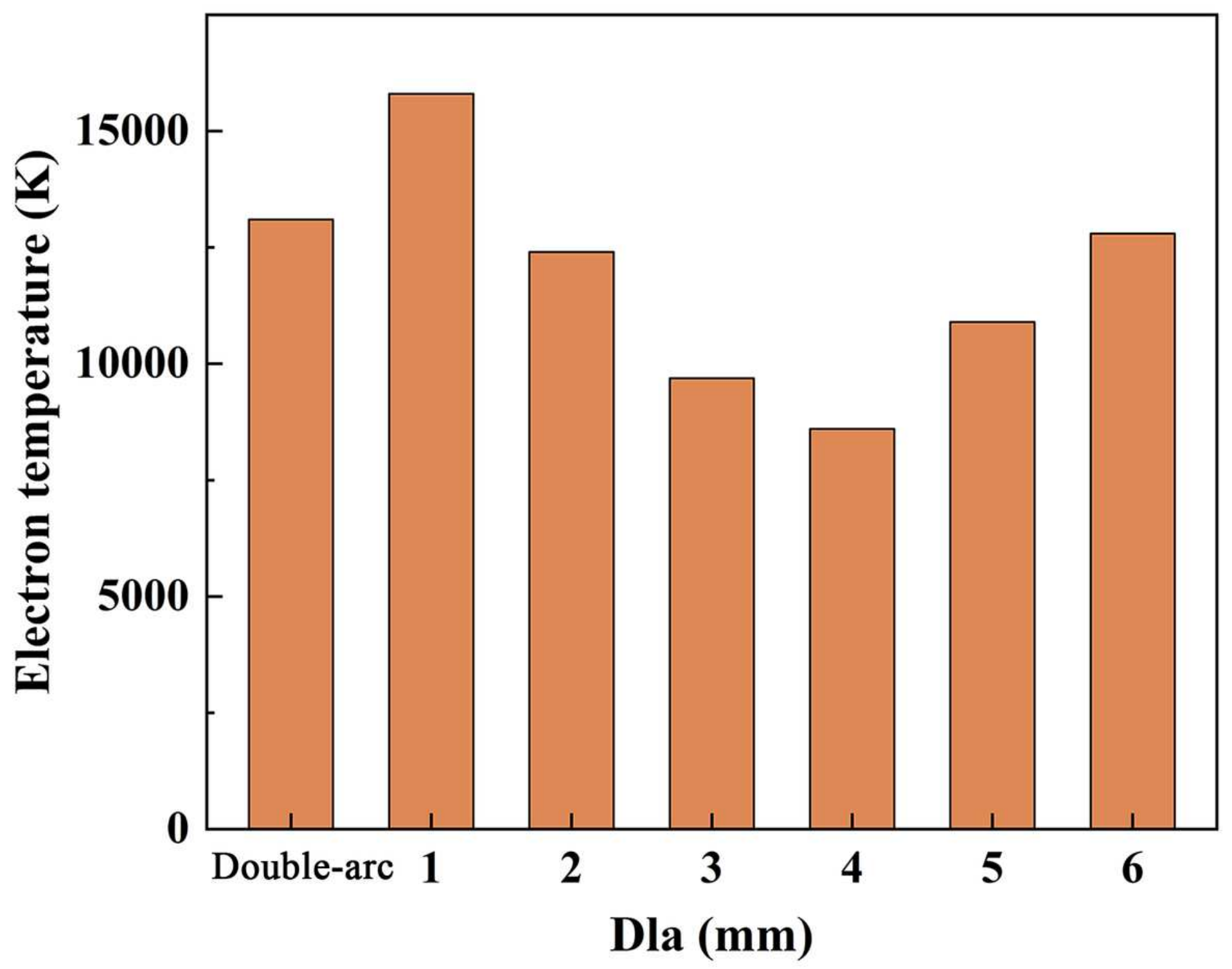

Figure 8

Electron temperature of double-arc plasma with different Dla

(a)

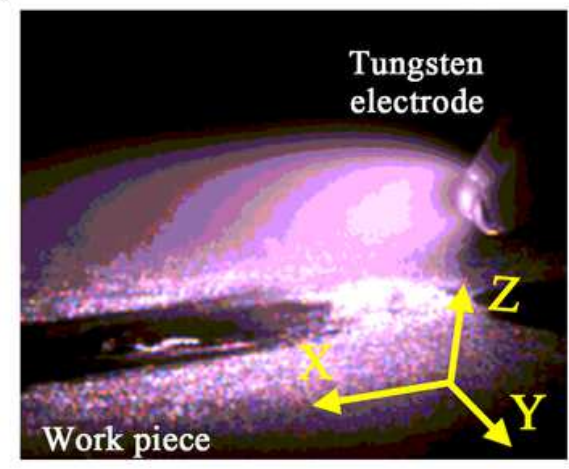

(b)

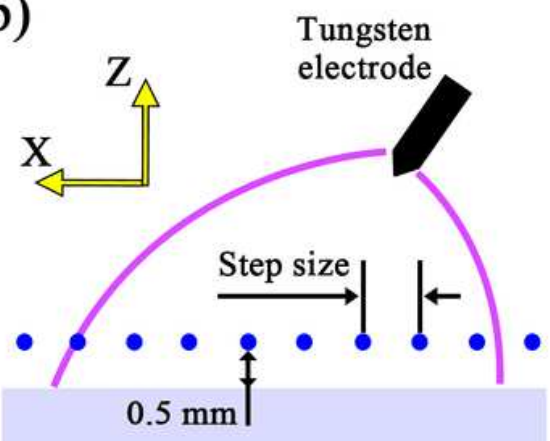

Work piece

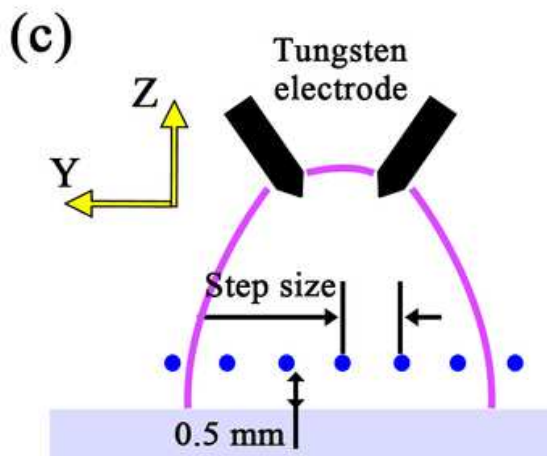

Work piece

Figure 9 

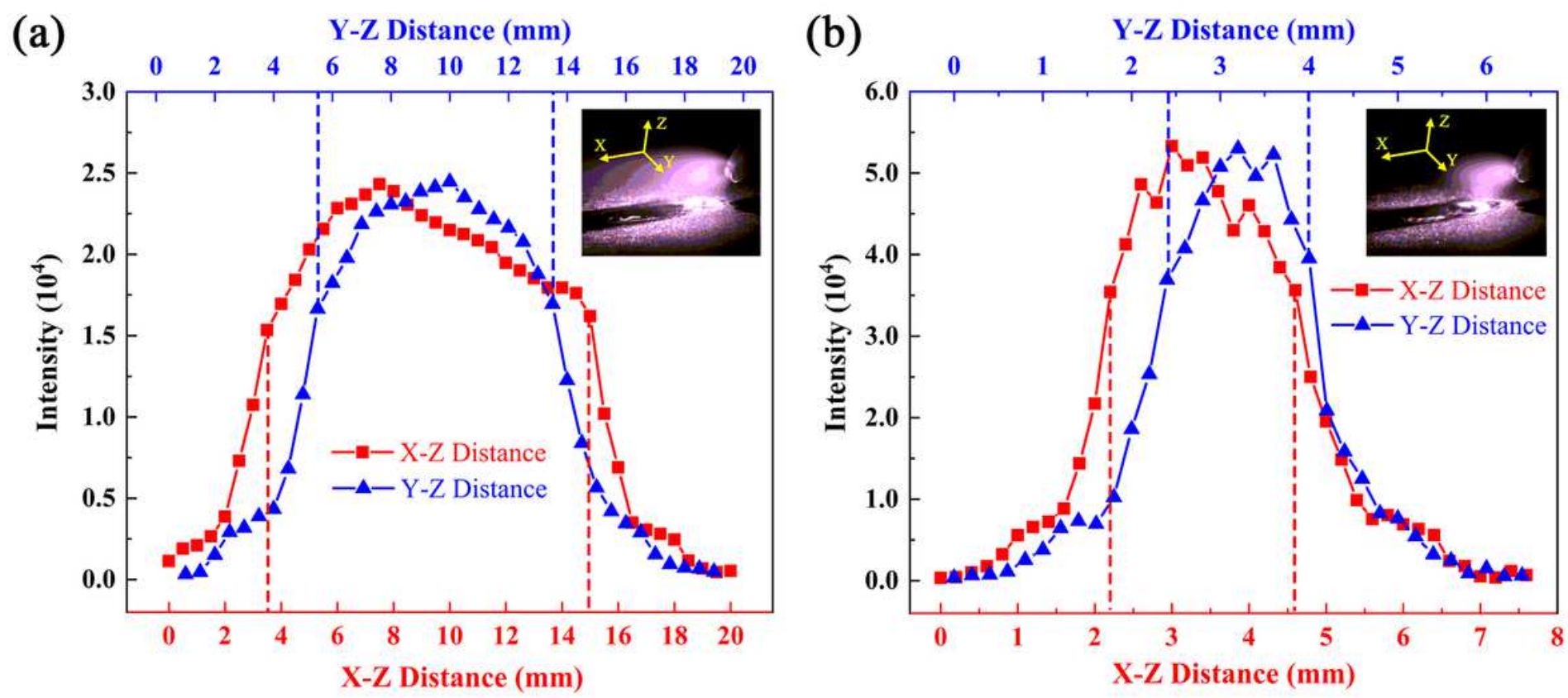

Figure 10

Spectral intensity of Mg I 516.732nm at various positions of double-arc (a) Traditional double-arc (b) Coupling double-arc
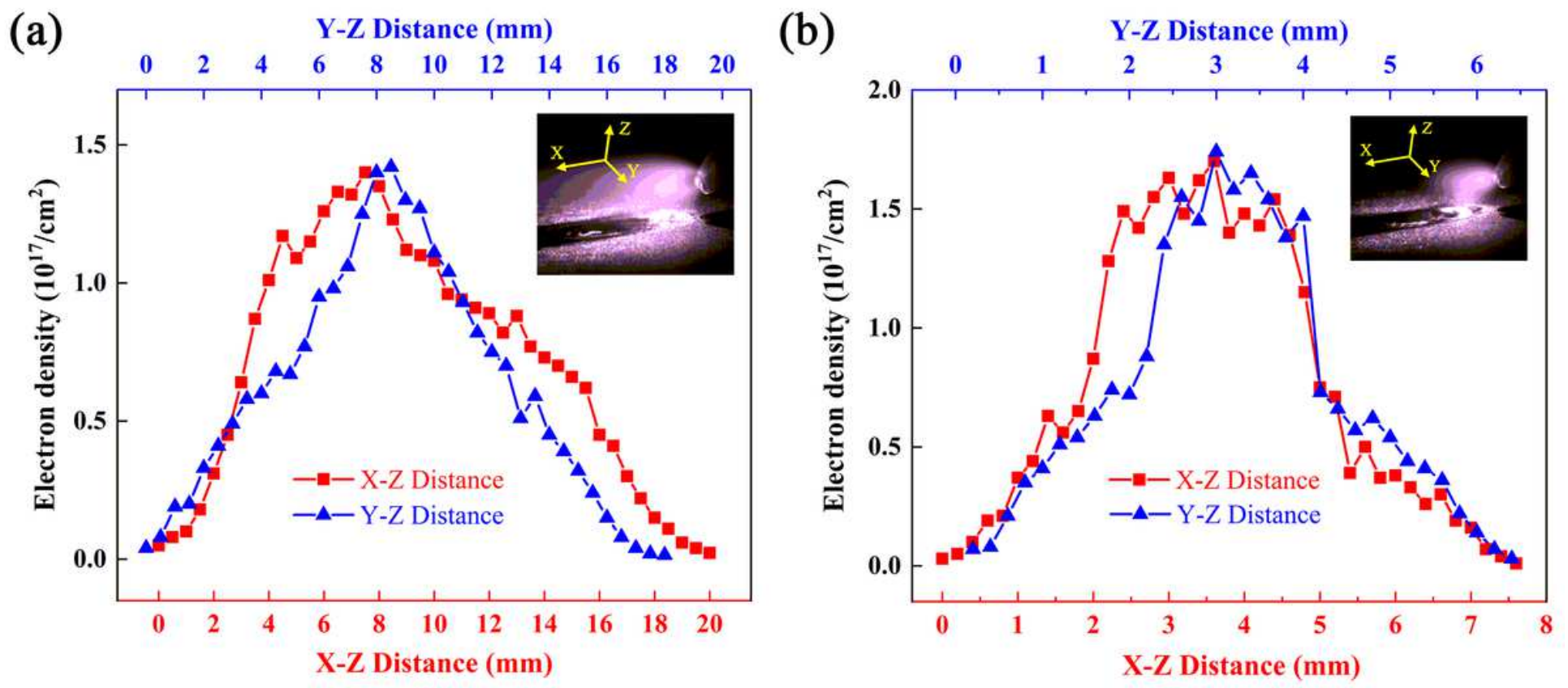

Figure 11

Electron density at various positions of arc (a) Traditional double-arc (b) Coupling double-arc 
(a)

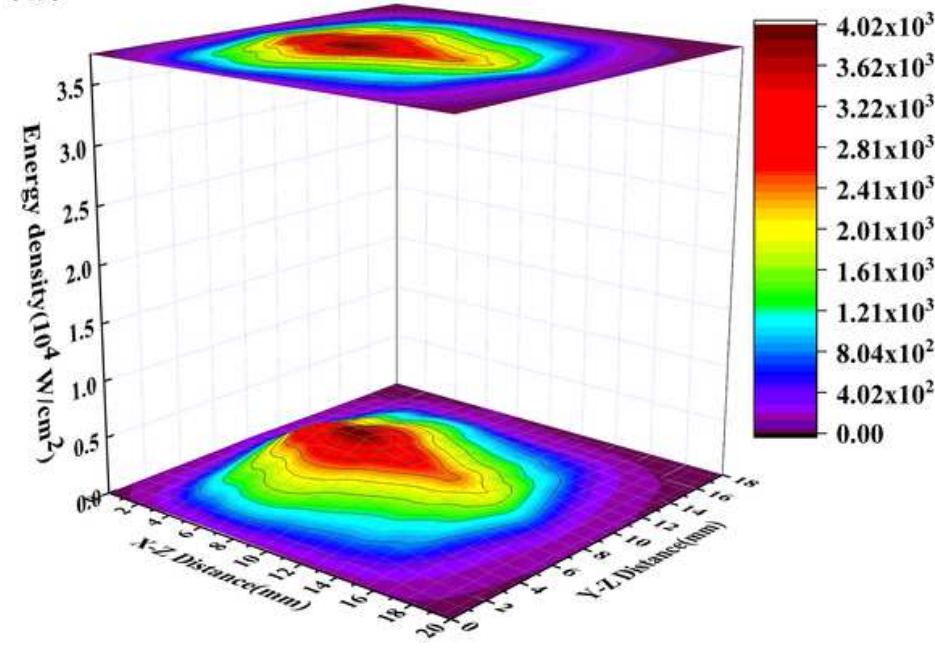

(b)

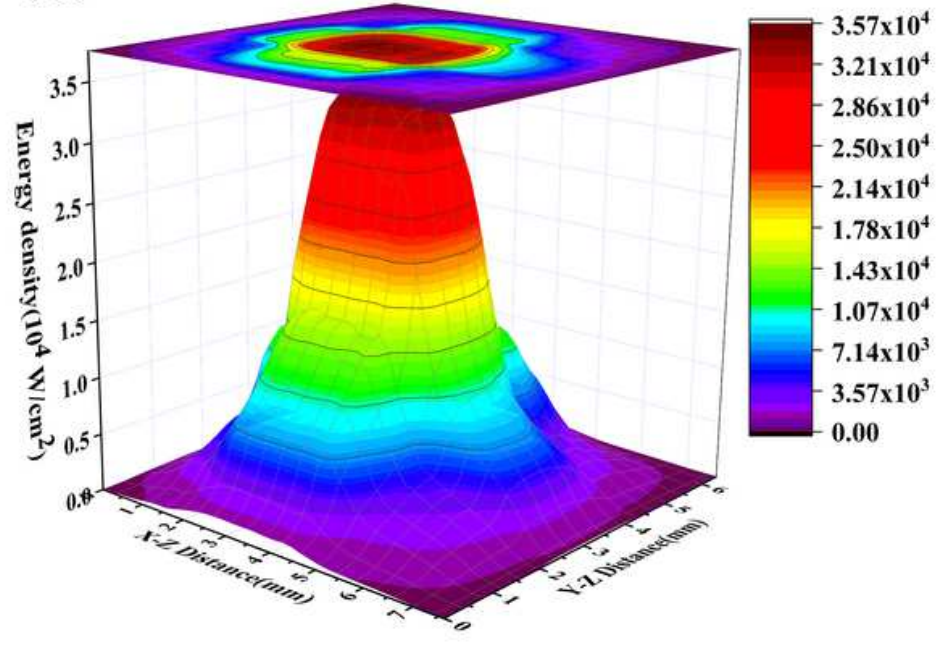

Figure 12

Spatial distribution of energy density of double-arc (a) Traditional double-arc (b) Coupling double-arc 
(a)

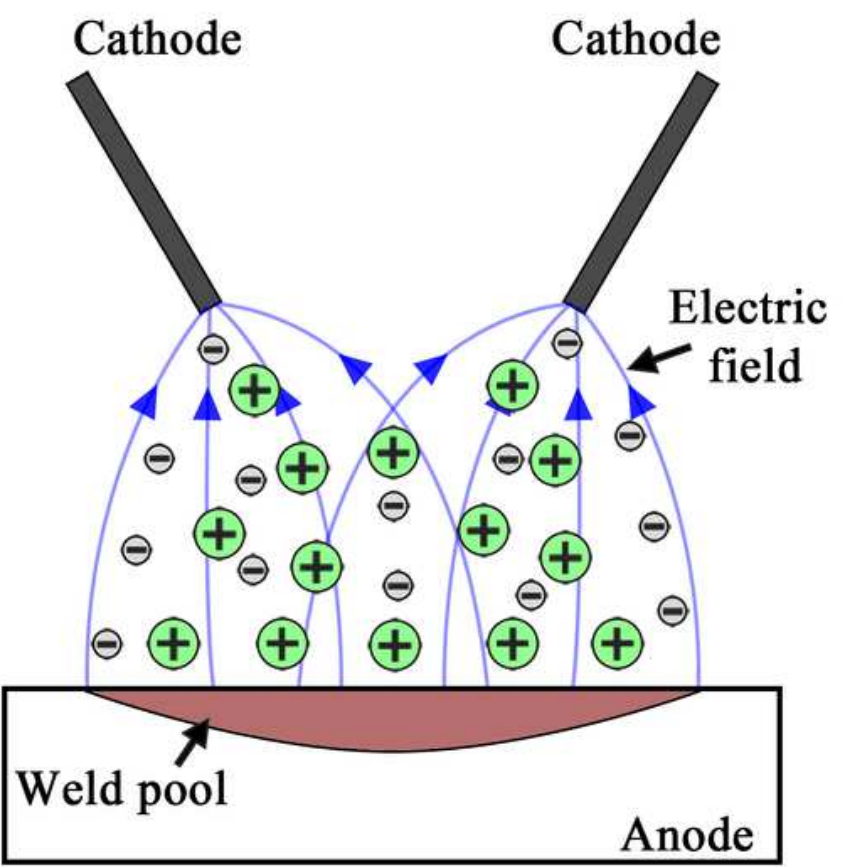

(c)

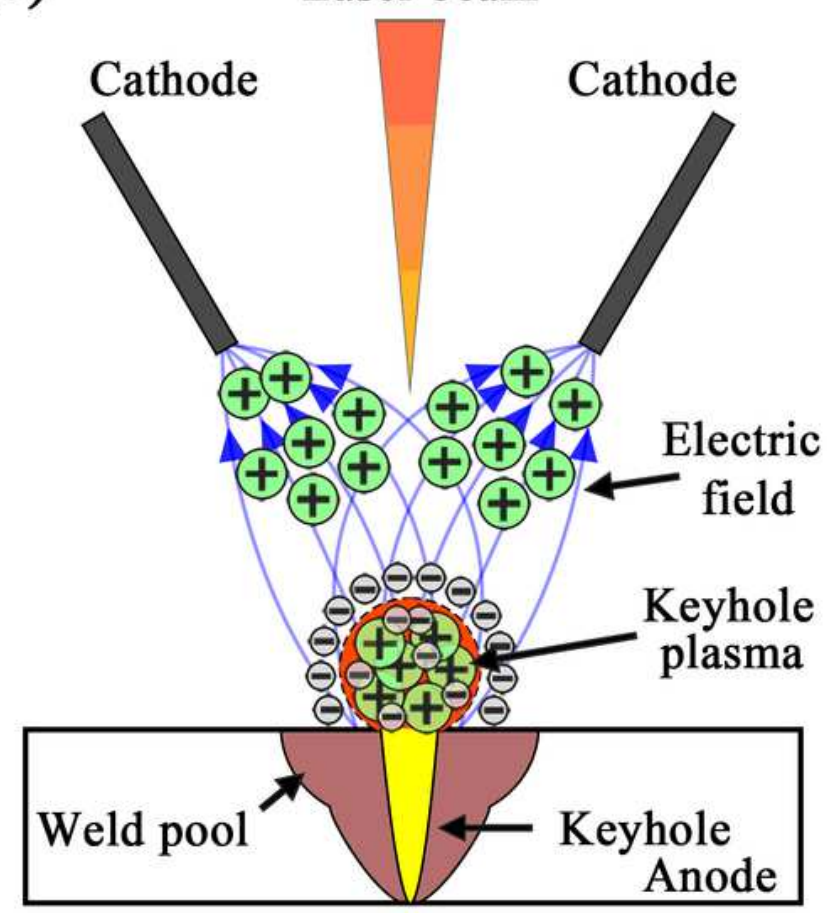

(b) Laser beam

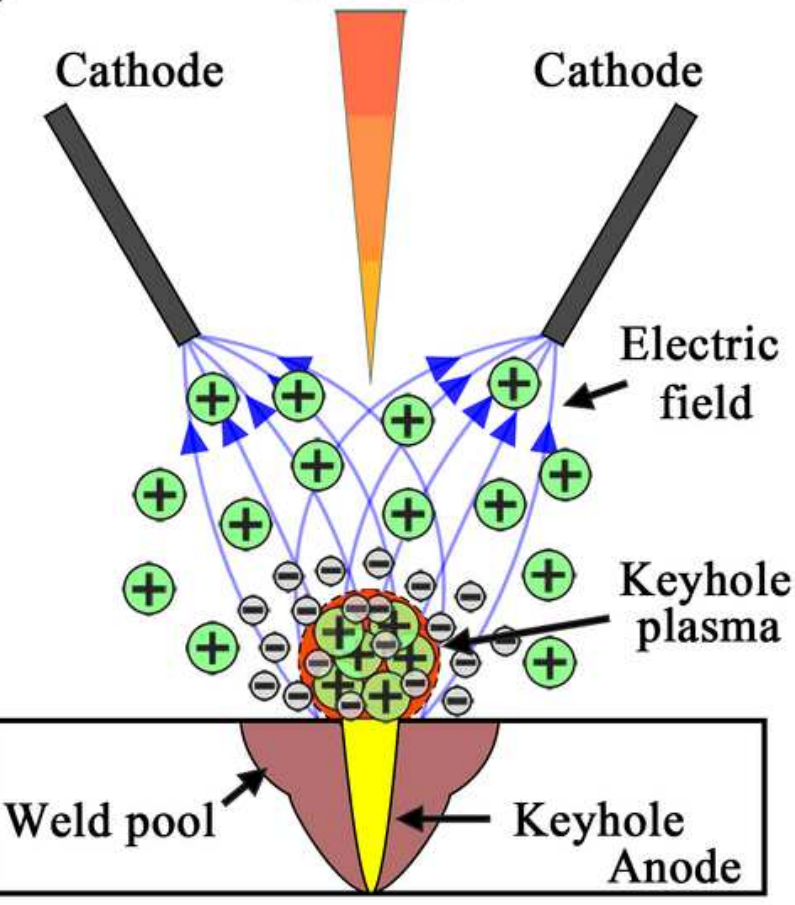

(d)

Laser beam

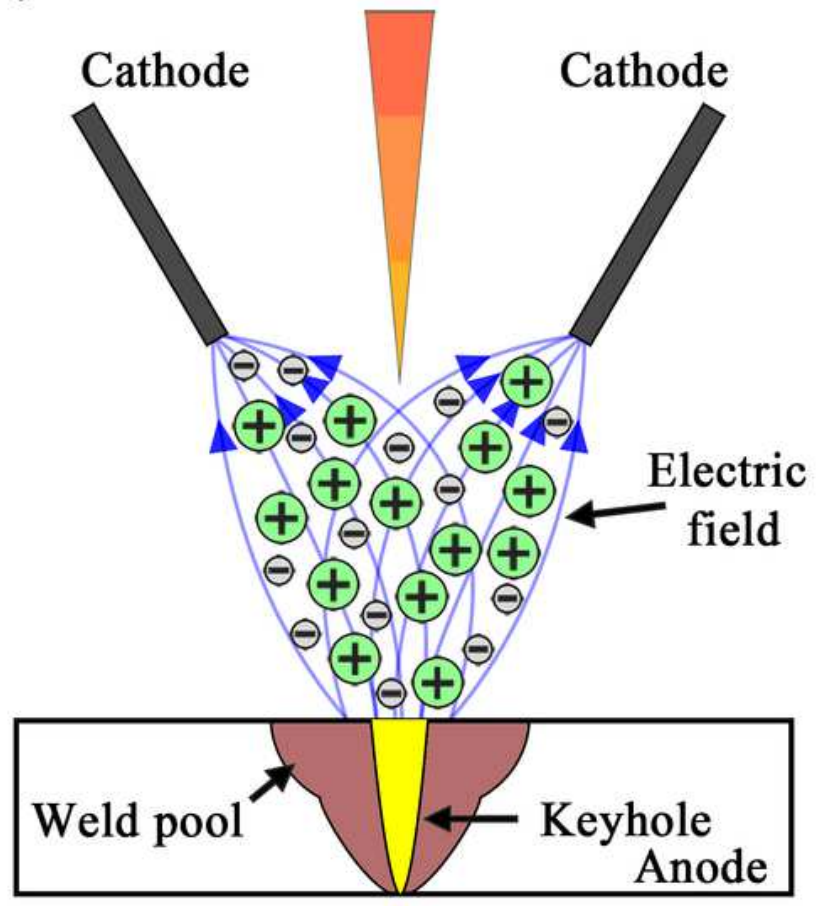

Figure 13

Schematic diagram of particle migration in double-arc induced by laser (a) Double-arc free discharge stage (b) Laser-induced initial stage (c) Laser-induced contraction stage (d) Laser-induced stable stage 
(a) Arc1

Arc2 Cathode

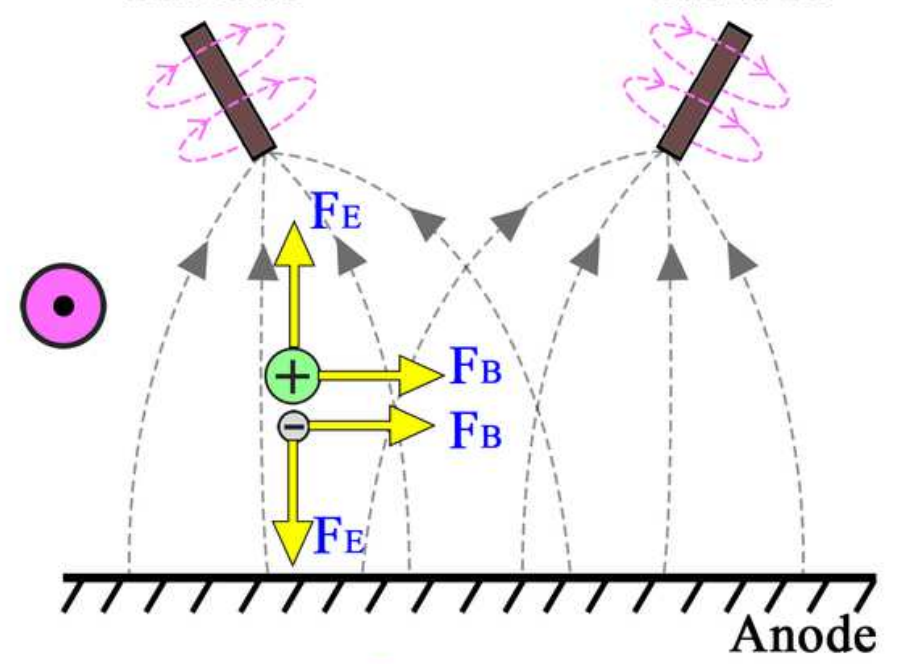

(b) Arc1 Arc2 Cathode Laser beam Cathode

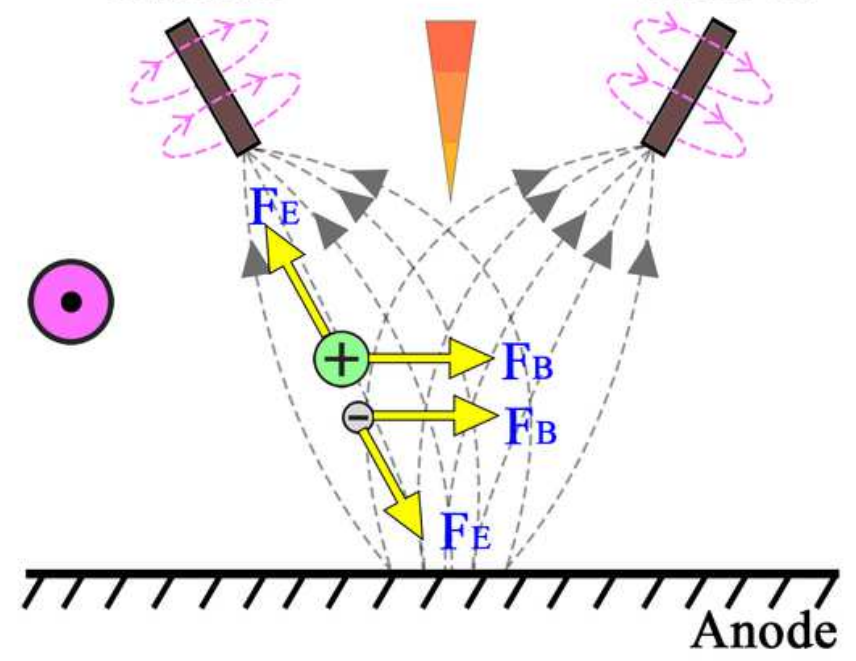

- Magnetic field direction produced by Arc 2

Figure 14

Schematic diagram of particle force (a) Traditional double-arc (b) Coupling double-arc 